\title{
Aggregating CL-Signatures Revisited: Extended Functionality and Better Efficiency*
}

\author{
Kwangsu Lee ${ }^{\dagger} \quad$ Dong Hoon Lee Moti Yung $^{\ddagger}$
}

\begin{abstract}
Aggregate signature is public-key signature that allows anyone to aggregate different signatures generated by different signers on different messages into a short (called aggregate) signature. The notion has many applications where compressing the signature space is important: secure routing protocols, compressed certificate chain signature, software module authentications, and secure high-scale repositories and logs for financial transactions. In spite of its importance, the state of the art of the primitive is that it has not been easy to devise a suitable aggregate signature scheme that satisfies the conditions of real applications, with reasonable parameters: short public key size, short aggregate signatures size, and efficient aggregate signing/verification. In this paper, we propose aggregate signature schemes based on the Camenisch-Lysyanskaya (CL) signature scheme (Crypto 2004) whose security is reduced to that of CL signature which substantially improve efficiency conditions for real applications.

- We first propose an efficient sequential aggregate signature scheme with the shortest size public key, to date, and very efficient aggregate verification requiring only a constant number of pairing operations and $l$ number of exponentiations ( $l$ being the number of signers).

- Next, we propose an efficient synchronized aggregate signature scheme with a very short public key size, and with the shortest (to date) size of aggregate signatures among synchronized aggregate signature schemes. Signing and aggregate verification are very efficient: they take constant number of pairing operations and $l$ number of exponentiations, as well.

- Finally, we introduce a new notion of aggregate signature named combined aggregate signature that allows a signer to dynamically use two modes of aggregation "sequential" and "synchronized," employing the same private/public key. We also present an efficient combined aggregate signature based on our previous two aggregate signature schemes. This combined-mode scheme allows for application flexibility depending on real world scenario: For example, it can be used sequentially to sign incrementally generated legal documents, and synchronously to aggregate the end-of-day logs of all branches of an institute into a single location with a single aggregate signature.
\end{abstract}

Keywords: Public-key signature, Aggregate information applications, Aggregate signature, CL signature, Bilinear maps.

\footnotetext{
*This is the extended and full version of the FC 2013 paper [25].

${ }^{\dagger}$ Korea University, Korea. Email: guspin@korea.ac.kr. This work was partially done at Columbia University.

$¥$ Korea University, Korea. Email: donghlee@korea. ac. kr.

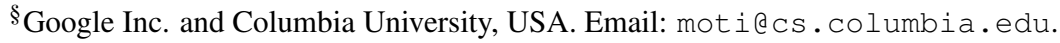




\section{Introduction}

Public-key signature (PKS) is a central cryptographic primitive with numerous applications. However, constructing a PKS scheme that is efficient, secure, and flexible enough for a range of possible applications is not easy. Among such schemes, CL signature, proposed by Camenisch and Lysyanskaya [17], is one of the pairing-based signature schemes [11, 14, 17, 35] that satisfies these conditions. It was widely used as a basic component in various cryptosystems such as anonymous credential systems, group signature, RFID encryption, batch verification signature, ring signature [2, 3, 7, 16, 17], as well as in aggregate signature [33].

Pubic-key aggregate signature (PKAS), introduced by Boneh, Gentry, Lynn, and Shacham [13], is a special type of PKS that enables anyone to aggregate different signatures generated by different signers on different messages into a short aggregate signature. Boneh et al. proposed the first full aggregate signature scheme in bilinear groups and proved its security in the random oracle model under the CDH assumption. After the introduction of aggregate signatures, various types of aggregate signatures such as sequential aggregate signatures [8, 9, 15, 20, 23, 27, 29, 32] and synchronized aggregate signatures [1, 22] were proposed. PKAS has numerous applications. In network and infrastructure: secure routing protocols, public-key infrastructure systems (signing certificate chains), sensor network systems, proxy signatures, as well as in applications: dynamically changing document composition (in particular, secure updating of software modules), secure transaction signing, secure work flow, and secure logs and repositories [1,9, 10, 13]. In all these applications, compressing the space consumed by signatures is the major advantage. Note that in the area of financial transactions, in particular, logs and repositories are very large due to regulatory requirements to hold records for long time periods. The effect of compressing signatures by aggregation increases with the number of data items; thus it is quite plausible that the financial sector may find variations of aggregate signature most useful.

Though PKAS can reduce the size of signers' signatures by using the aggregation technique, it cannot reduce the size of signers' public keys since the public keys are not aggregated. Thus, the total information the verifier needs to access is still proportional to the number of signers in the aggregate signature, since the verifier should retrieve all public keys of signers from a certificate storage. Therefore, it is very important to reduce the size of public keys. An ideal solution for this problem is to use identity-based aggregate signature (IBAS) that represents the public key of a signer as an identity string. However, IBAS requires a trust structure different from public key infrastructure, namely, the existence of an additional trusted authority, (the current IBAS schemes are in [9, 22, 23] and are all secure in the random oracle model.) To construct a PKAS scheme with short public keys, Schröder proposed a sequential aggregate signature scheme with short public keys based on the CL signature scheme [33]. In the scheme of Schröder, the public key consists of two group elements and the aggregate signature consists of four group elements, but the aggregate verification algorithm requires $l$ pairing operations and $l$ exponentiations where $l$ is the number of signers in the aggregate signature. Therefore, this work, while nicely pointing at the CL signature as a source of efficiency for the context of aggregate signatures, still leaves out desired properties to build upon while exploiting the flexibility of the CL signature: can we make the public key shorter? can we require substantially less work in verification? and, can we build other modes of aggregate signatures? While asking such questions, we revisit the subject of aggregate signature based on CL signatures.

\subsection{Our Contributions}

In this paper, we indeed solve the problem of constructing a PKAS scheme that has short public keys, short aggregate signatures, and an efficient aggregate verification algorithm. 
Efficient Sequential Aggregate Signature. We first propose an efficient sequential aggregate signature scheme based on the CL signature scheme and prove its security based on that of CL signature (i.e., the LRSW assumption) without random oracles. A sequential aggregate signature assumes that the aggregation mode is done in linear order: signed message after signed message. In this scheme, the public key consists of just one group element and the aggregate signature consists of just three group element. The size of the public key is the shortest among all sequential aggregate schemes to date (except IBAS schemes). The aggregate verification algorithm of our scheme is quite efficient since it just requires five pairing operations and $l$ exponentiations (or multi-exponentiations). Therefore our scheme simultaneously satisfies the conditions of short public keys, short aggregate signatures, and efficient aggregate verification.

Efficient Synchronized Aggregate Signature. Next, we propose an efficient synchronized aggregate signature scheme based on the CL signature scheme and prove its security based on the CL signature security in the random oracle model (the random oracle can be removed if the number of messages is restricted to be polynomial). Synchronized aggregate signature is a mode where the signers of messages to be aggregated are synchronized, but aggregation can take any order. In this scheme, the public key consists of just one group element and the aggregate signature consists of one group element and one integer. The size of the aggregate signature is the shortest among all synchronized aggregate signature schemes to date. The aggregate verification algorithm of this scheme is also quite efficient since it just requires three pairing operations and $l$ exponentiations (or multi-exponentiations).

New Combined Aggregate Signature. Finally, we show that our two aggregate signature schemes can be combined to give a new notion of combined aggregate signature: A scheme which supports, both, sequential aggregation or synchronized aggregation, since the public key and the private key of two schemes are the same. This property can increase the utility and flexibility of the suggested scheme(s). We define the formal definition of combined aggregate signature and present an efficient scheme based on our two previous aggregate signature schemes. The security of this scheme is also based on the security of the CL signature.

\subsection{Our Technique}

Technically speaking, in order to construct our schemes from the CL signature scheme, we employ two techniques: the first one is an adaptation of the "randomness re-use" technique of Lu et al. [28], and the second one is a newly devised "public key sharing" technique. Our "public key sharing" technique distributes the element $Y$ of the public key among all signers by placing the public key element $Y$ of the CL signature into the public parameters. In this case, the private key and the public key of a signer are $x$ and $X=g^{x}$ instead of $x, y$ and $X=g^{x}, Y=g^{y}$ respectively. The signer can then generate the original CL signature as $\sigma=\left(A=g^{r}, B=Y^{r}, C=A^{x} B^{x M}\right)$. Furthermore, the signer can also generate a sequential aggregate signature as $\sigma_{\Sigma}=\left(A=g^{r}, B=Y^{r}, C=A^{\sum x_{i}} B^{\sum x_{i} M_{i}}\right)$ since he only needs to aggregate the elements related to the public keys $\left\{X_{i}\right\}$ by using the "randomness re-use" technique. To construct a synchronized aggregate signature scheme from the sequential aggregate signature scheme, we force all signers to use the same elements $A$ and $B$ by using the synchronized time period information. That is, a signer first sets $A=H(0 \| w)$ and $B=H(1 \| w)$ where $H$ is a hash function and $w$ is a time period, and he generates a synchronized aggregate signature as $\sigma_{\Sigma}=\left(C=A^{\sum x_{i}} B^{\sum x_{i} M_{i}}, w\right)$.

\subsection{Related Work}

Given the importance of aggregation to saving signature space, much work has been invested in the various notions allowing aggregation. 
Full Aggregation. The notion of public-key aggregate signature (PKAS) was introduced by Boneh, Gentry, Lynn, and Shacham [13]. They proposed the first PKAS scheme in bilinear groups that supports full aggregation such that anyone can freely aggregate different signatures signed by different signers on different messages into a short aggregate signature. The PKAS scheme of Boneh et al. [13] requires $l$ number of pairing operations in the aggregate verification algorithm where $l$ is the number of signers in the aggregate signature. Bellare et al. [5] modified the PKAS scheme of Boneh et al. to remove the restriction such that the message should be different by hashing a message with the public key of a signer. Subsequent to our work, Hohenberger, Sahai, and Waters [24] proposed an identity-based full aggregate signature scheme based on candidate multilinear maps of Garg et al. [21].

Sequential Aggregation. The concept of sequential aggregate signature was introduced by Lysyanskaya, Micali, Reyzin, and Shacham [29]. In sequential aggregate signature, a signer can generate an aggregate signature by adding his signature to the previous aggregate signature that was received from a previous signer. Lysyanskaya et al. [29] proposed a sequential PKAS scheme using certified trapdoor permutations, and they proved its security in random oracle models. Neven [32] proposed a sequential PKAS scheme that reduces not only the size of signatures but also the size of total information that is transmitted. Boldyreva et al. [9] proposed an identity-based sequential aggregate signature (IBSAS) scheme (in the trust model of identity-based schemes with a trusted private keys authority), in bilinear groups and proved its security in the random oracle model under an interactive assumption. Recently, Gerbush et al. [23] showed that a modified IBSAS scheme of Boldyreva et al. in composite order bilinear groups can be secure in the random oracle model under static assumptions.

The first sequential PKAS scheme without random oracles was proposed by Lu et al. [28]. They constructed a sequential PKAS scheme based on the PKS scheme of Waters and proved its security without random oracles under the $\mathrm{CDH}$ assumption. However, this sequential PKAS scheme has a disadvantage such that the size of public keys is very long. To reduce the size of pubic keys in PKAS schemes, Schröder proposed the CL signature based scheme discussed above [33]. Recently, Lee et al. [26, 27] proposed an efficient sequential PKAS scheme with short public keys and proved its security without random oracles under static assumptions.

In sequential PKAS schemes, a signer generally should verify the validity of the previous aggregate signature (the aggregate-so-far) handed to him from a previous signer before he adds his signature into the aggregate signature. To verify the previous aggregate signature, the signer should retrieve all public keys of previous signers and should run the aggregate verification algorithm. Thus verifying the previous aggregate signature is the most expensive operation in the aggregate signing algorithm. To solve this problem, sequential PKAS schemes that do not require to verify the previous aggregate signature were proposed [15, 20].

Synchronized Aggregation. The concept of synchronized aggregate signature was introduced by Gentry and Ramzan [22]. In synchronized aggregate signature, all signers have synchronized time information and individual signatures generated by different signers within the same time period can be aggregated into a short aggregate signature. They proposed an identity-based synchronized aggregate signature scheme in bilinear groups and proved its security in the random oracle model under the CDH assumption. Ahn et al. [1] proposed an efficient synchronized PKAS scheme based on the PKS scheme of Hohenberger and Waters and proved its security without random oracles under the $\mathrm{CDH}$ assumption.

Interactive Aggregation. Interactive aggregate signature is aggregate signature such that a signer generates an aggregate signature after having interactive communications with other signers through a broadcast channel. Bellare and Neven [6] proposed an identity-based multi-signature scheme in the random oracle model under the RSA assumption, and Bagherzandi and Jareki [4] proposed an identity-based aggregate signature 
scheme and proved its security in the random oracle model under the RSA assumption. However, the interactive communications between signers are expensive (signing becomes a protocol among parties), and the heavy message transmission between signers may eliminate some of the advantage of signature aggregation.

\section{Preliminaries}

In this section, we first define the public key signature and its security model. Next, we define bilinear groups, and introduce the LRSW assumption which is associated with the security of the CL signature scheme, which is, then, presented as well.

\subsection{Public Key Signature}

A public key signature (PKS) scheme consists of three PPT algorithms KeyGen, Sign, and Verify, which are defined as follows: The key generation algorithm $\operatorname{KeyGen}\left(1^{\lambda}\right)$ takes as input a security parameter $1^{\lambda}$, and outputs a public key $P K$ and a private key $S K$. The signing algorithm $\operatorname{Sign}(M, S K)$ takes as input a message $M$ and a private key $S K$, and outputs a signature $\sigma$. The verification algorithm $\operatorname{Verify}(\sigma, M, P K)$ takes as input a signature $\sigma$, a message $M$, and a public key $P K$, and outputs either 1 or 0 depending on the validity of the signature.

The correctness requirement is that for any $(P K, S K)$ output by KeyGen and any $M \in \mathcal{M}$, we have that $\operatorname{Verify}(\operatorname{Sign}(M, S K), M, P K)=1$. We can relax this notion to require that the verification is correct with overwhelming probability over all the randomness of the experiment.

The security notion of existential unforgeability under a chosen message attack is defined in terms of the following experiment between a challenger $\mathcal{C}$ and a PPT adversary $\mathcal{A}: \mathcal{C}$ first generates a key pair $(P K, S K)$ by running KeyGen, and gives $P K$ to $\mathcal{A}$. Then $\mathcal{A}$, adaptively and polynomially many times, requests a signature query on a message $M$ under the challenge public key $P K$, and receives a signature $\sigma$. Finally, $\mathcal{A}$ outputs a forged signature $\sigma^{*}$ on a message $M^{*}$. $\mathcal{C}$ then outputs 1 if the forged signature satisfies the following two conditions, or outputs 0 otherwise: 1) $\operatorname{Verify}\left(\sigma^{*}, M^{*}, P K\right)=1$ and 2) $M^{*}$ was not queried by $\mathcal{A}$ to the signing oracle. The advantage of $\mathcal{A}$ is defined as $\operatorname{Adv}_{\mathcal{A}}^{P K S}=\operatorname{Pr}[\mathcal{C}=1]$ where the probability is taken over all the randomness of the experiment. A PKS scheme is existentially unforgeable under a chosen message attack if all PPT adversaries have at most a negligible advantage in the above experiment (for large enough security parameter).

\subsection{Bilinear Groups}

Let $\mathbb{G}$ and $\mathbb{G}_{T}$ be multiplicative cyclic groups of prime order $p$. Let $g$ be a generator of $\mathbb{G}$. The bilinear map $e: \mathbb{G} \times \mathbb{G} \rightarrow \mathbb{G}_{T}$ has the following properties:

1. Bilinearity: $\forall u, v \in \mathbb{G}$ and $\forall a, b \in \mathbb{Z}_{p}, e\left(u^{a}, v^{b}\right)=e(u, v)^{a b}$.

2. Non-degeneracy: $\exists g$ such that $e(g, g)$ has order $p$, that is, $e(g, g)$ is a generator of $\mathbb{G}_{T}$.

We say that $\mathbb{G}, \mathbb{G}_{T}$ are bilinear groups if the group operations in $\mathbb{G}$ and $\mathbb{G}_{T}$ as well as the bilinear map $e$ are all efficiently computable. 


\subsection{Complexity Assumption}

The security of our aggregate signature schemes is based on the following LRSW assumption. The LRSW assumption was introduced by Lysyanskaya et al. [30] and it is secure under the generic group model defined by Shoup [34] (and adapted to bilinear groups in [17]). We also define the one-time LRSW (OT-LRSW) assumption that is a static variant of the LRSW assumption.

Assumption 2.1 (LRSW). Let $\mathcal{G}$ be an algorithm that on input the security parameter $1^{\lambda}$, outputs the parameters for a bilinear group as $\left(p, \mathbb{G}, \mathbb{G}_{T}, e, g\right)$. Let $X, Y \in \mathbb{G}$ such that $X=g^{x}, Y=g^{y}$ for some $x, y \in \mathbb{Z}_{p}$. Let $O_{X, Y}(\cdot)$ be an oracle that on input a value $M \in \mathbb{Z}_{p}$ outputs a triple $\left(a, a^{y}, a^{x+M x y}\right)$ for a randomly chosen $a \in \mathbb{G}$. Then for all probabilistic polynomial time adversaries $\mathcal{A}$,

$$
\begin{aligned}
\operatorname{Pr} & {\left[\left(p, \mathbb{G}, \mathbb{G}_{T}, e, g\right) \leftarrow \mathcal{G}\left(1^{\lambda}\right), x \leftarrow \mathbb{Z}_{p}, y \leftarrow \mathbb{Z}_{p}, X=g^{x}, Y=g^{y},\right.} \\
& (M, a, b, c) \leftarrow \mathcal{A}^{O_{X, Y}(\cdot)}\left(p, \mathbb{G}, \mathbb{G}_{T}, e, g, X, Y\right): \\
& \left.M \notin Q \wedge M \in \mathbb{Z}_{p}^{*} \wedge a \in \mathbb{G} \wedge b=a^{y} \wedge c=a^{x+M x y}\right]<1 / \operatorname{poly}(\lambda)
\end{aligned}
$$

where $Q$ is the set of queries that $\mathcal{A}$ made to $O_{X, Y}(\cdot)$.

Assumption 2.2 (OT-LRSW). Let $\mathcal{G}$ be an algorithm that on input the security parameter $1^{\lambda}$, outputs the parameters for a bilinear group as $\left(p, \mathbb{G}, \mathbb{G}_{T}, e, g\right)$. Then for all probabilistic polynomial time adversaries $\mathcal{A}$,

$$
\begin{aligned}
& \operatorname{Pr}\left[\left(p, \mathbb{G}, \mathbb{G}_{T}, e, g\right) \leftarrow \mathcal{G}\left(1^{\lambda}\right), x \leftarrow \mathbb{Z}_{p}, y \leftarrow \mathbb{Z}_{p}, R \leftarrow \mathbb{Z}_{p}, d \leftarrow \mathbb{G},\right. \\
& \quad(M, a, b, c) \leftarrow \mathcal{A}\left(p, \mathbb{G}, \mathbb{G}_{T}, e, g, X=g^{x}, Y=g^{y}, R, d, d^{y}, d^{x+R x y}\right): \\
& \left.M \neq R \wedge M \in \mathbb{Z}_{p}^{*} \wedge a \in \mathbb{G} \wedge b=a^{y} \wedge c=a^{x+M x y}\right]<1 / \operatorname{poly}(\lambda) .
\end{aligned}
$$

\subsection{The CL Signature Scheme}

The CL signature scheme is a public-key signature scheme that was proposed by Camenisch and Lysyanskaya [17] and the security was proven without random oracles under the LRSW assumption. Although the security of the CL signature scheme is based on this interactive assumption, it is flexible and widely used for the constructions of various cryptosystems such as anonymous credentials, group signatures, ring signatures, batch verification, and aggregate signatures [7, 16, 17, 30, 33] (this is so, perhaps due to its relatively elegant and simple algebraic structure).

PKS.KeyGen $\left(1^{\lambda}\right)$ : The key generation algorithm first generates the bilinear groups $\mathbb{G}, \mathbb{G}_{T}$ of prime order $p$ of bit size $\Theta(\lambda)$. Let $g$ be the generator of $\mathbb{G}$. It selects two random exponents $x, y \in \mathbb{Z}_{p}$ and sets $X=g^{x}, Y=g^{y}$. It outputs a private key as $S K=(x, y)$ and a public key as $P K=\left(p, \mathbb{G}_{,} \mathbb{G}_{T}, e, g, X, Y\right)$.

PKS.Sign $(M, S K)$ : The signing algorithm takes as input a message $M \in \mathbb{Z}_{p}^{*}$ and a private key $S K=(x, y)$. It selects a random element $A \in \mathbb{G}$ and computes $B=A^{y}, C=A^{x} B^{x M}$. It outputs a signature as $\sigma=(A, B, C)$.

PKS.Verify $(\sigma, M, P K)$ : The verification algorithm takes as input a signature $\sigma=(A, B, C)$ on a message $M \in \mathbb{Z}_{p}^{*}$ under a public key $P K=\left(p, \mathbb{G}, \mathbb{G}_{T}, e, g, X, Y\right)$. It verifies that $e(A, Y) \stackrel{?}{=} e(B, g)$ and $e(C, g) \stackrel{?}{=}$ $e(A, X) \cdot e(B, X)^{M}$. If these equations hold, then it outputs 1 . Otherwise, it outputs 0 .

Theorem 2.3 ( [17]). The CL signature scheme is existentially unforgeable under a chosen message attack if the LRSW assumption holds. 


\section{Sequential Aggregate Signature}

In this section, we first define the sequential aggregate signature and its security model. After that, we propose an efficient sequential aggregate signature scheme based on the CL signature scheme, and prove its security under the LRSW assumption.

\subsection{Definitions}

Sequential aggregate signature (SeqAS) is a special type of public-key aggregate signature (PKAS) that allows each signer to sequentially add his signature on a different message to the aggregate signature [29]. That is, a signer with an index $i$ receives an aggregate signature $\sigma_{\Sigma}^{\prime}$ from the signer of an index $i-1$, and he generates a new aggregate signature $\sigma_{\Sigma}$ by aggregating his signature on a message $M$ to the received aggregate signature. The resulting aggregate signature has the same size of the previous aggregate signature.

Definition 3.1 (Sequential Aggregate Signature). A sequential aggregate signature (SeqAS) scheme consists of four PPT algorithms Setup, KeyGen, AggSign, and AggVerify, which are defined as follows:

$\operatorname{Setup}\left(1^{\lambda}\right)$. The setup algorithm takes as input a security parameter $1^{\lambda}$ and outputs public parameters $P P$.

$\operatorname{Key} \operatorname{Gen}(P P)$. The key generation algorithm takes as input the public parameters $P P$, and outputs a public key $P K$ and a private key $S K$.

$\operatorname{AggSign}\left(\sigma_{\Sigma}^{\prime}, \mathbf{M}, \mathbf{P K}, M, S K, P P\right)$. The aggregate signing algorithm takes as input an aggregate-so-far $\sigma_{\Sigma}^{\prime}$ on messages $\mathbf{M}=\left(M_{1}, \ldots, M_{k}\right)$ under public keys $\mathbf{P K}=\left(P K_{1}, \ldots, P K_{k}\right)$, a message $M$, and a private key $S K$ with $P P$, and outputs a new aggregate signature $\sigma_{\Sigma}$.

$\operatorname{AggVerify}\left(\sigma_{\Sigma}, \mathbf{M}, \mathbf{P K}, P P\right)$. The aggregate verification algorithm takes as input an aggregate signature $\sigma_{\Sigma}$ on messages $\mathbf{M}=\left(M_{1}, \ldots, M_{l}\right)$ under public keys $\mathbf{P K}=\left(P K_{1}, \ldots, P K_{l}\right)$ and the public parameters $P P$, and outputs either 1 or 0 depending on the validity of the aggregate signature.

The correctness requirement is that for each PP output by Setup, for all $(P K, S K)$ output by KeyGen, any $M$, we have that AggVerify $\left(\boldsymbol{A g g S i g n}\left(\sigma_{\Sigma}^{\prime}, \mathbf{M}^{\prime}, \mathbf{P} \mathbf{K}^{\prime}, M, S K, P K, P P\right), \mathbf{M}^{\prime}\left\|M, \mathbf{P K} \mathbf{K}^{\prime}\right\| P K, P P\right)=1$ where $\sigma_{\Sigma}^{\prime}$ is a valid aggregate-so-far signature on messages $\mathbf{M}^{\prime}$ under public keys $\mathbf{P} \mathbf{K}^{\prime}$.

The security model of SeqAS was introduced by Lysyanskaya et al. [29]. In this paper, we follow the security model that was proposed by Lu et al. [28]. The security model of Lu et al. is a more restricted model that requires the adversary to correctly generate other signers' public keys and private keys except the challenge signer's key. To ensure the correct generation of public keys and private keys, the adversary should submit the corresponding private keys of the public keys to the challenger before using the public keys. A realistic solution of this is for the signer to prove that he knows the corresponding private key of the public key by using zero-knowledge proofs when he requests the certification of his public key.

In the security model of SeqAS, the public parameters and the challenge public key $P K^{*}$ are given to the adversary. The adversary can request the certification of a public key through a certification query by providing a public key and a private key. It also can request a sequential aggregate signature on a message under the challenge public key by providing an aggregate-so-far that was generated from the certified public keys. Finally, it outputs a forged aggregate signature $\sigma_{\Sigma}^{*}$. The adversary breaks the SeqAS scheme if the forged sequential aggregate signature is valid and non-trivial.

Definition 3.2 (Unforgeability). The security notion of existential unforgeability under a chosen message attack is defined in terms of the following experiment between a challenger $\mathcal{C}$ and a PPT adversary $\mathcal{A}$ : 
1. Setup: $\mathcal{C}$ first initializes a key-pair list KeyList as empty. Next, it runs Setup to obtain public parameters $P P$ and KeyGen to obtain a key pair $(P K, S K)$, and gives $P K$ to $\mathcal{A}$.

2. Certification Query: $\mathcal{A}$ adaptively requests the certification of a public key by providing a key pair $(P K, S K)$. Then $\mathcal{C}$ adds the key pair $(P K, S K)$ to KeyList if the key pair is a valid one.

3. Signature Query: A adaptively requests a sequential aggregate signature (by providing an aggregateso-far $\sigma_{\Sigma}^{\prime}$ on messages $\mathbf{M}^{\prime}$ under public keys $\left.\mathbf{P} \mathbf{K}^{\prime}\right)$, on a message $M$ to sign under the challenge public key $P K$, and receives a sequential aggregate signature $\sigma_{\Sigma}$.

4. Output: Finally (after a sequence of the above queries), $\mathcal{A}$ outputs a forged sequential aggregate signature $\sigma_{\Sigma}^{*}$ on messages $\mathbf{M}^{*}$ under public keys $\mathbf{P} \mathbf{K}^{*} . \mathcal{C}$ outputs 1 if the forged signature satisfies the following three conditions, or outputs 0 otherwise: 1) AggVerify $\left.\left(\sigma_{\Sigma}^{*}, \mathbf{M}^{*}, \mathbf{P K} \mathbf{K}^{*}, P P\right)=1,2\right)$ The challenge public key PK must exist in $\mathbf{P} \mathbf{K}^{*}$ and each public key in $\mathbf{P} \mathbf{K}^{*}$ except the challenge public key must be in KeyList, and 3) The corresponding message $M$ in $\mathbf{M}^{*}$ of the challenge public key PK must not have been queried by $\mathcal{A}$ to the sequential aggregate signing oracle.

The advantage of $\mathcal{A}$ is defined as $\boldsymbol{A d} \boldsymbol{v}_{\mathcal{A}}^{\text {SeqAS }}=\operatorname{Pr}[\mathcal{C}=1]$ where the probability is taken over all the randomness of the experiment. A SeqAS scheme is existentially unforgeable under a chosen message attack if all PPT adversaries have at most a negligible advantage (for large enough security parameter) in the above experiment.

\subsection{Design Principle}

We first describe the design idea of our SeqAS scheme. To construct a SeqAS scheme, we use the "public key sharing" technique such that the element $Y$ in the public key of the original CL signature scheme can be shared with all signers. The modified CL signature scheme that shares the element $Y$ of the public key is described as follows: The setup algorithm publishes the public parameters that contain the description of bilinear groups and an element $Y$. Each signer generates a private key $x \in \mathbb{Z}_{p}$ and a public key $X=g^{x}$. A signer who has the private key $x$ of the public key $X$ can generate an original CL signature $\sigma=(A, B, C)$ on a message $M$ just using the private key $x$ and a random $r$ as $A=g^{r}, B=Y^{r}$, and $C=A^{x} B^{x M}$ since the element $Y$ is given in the public parameters. This modified CL signature scheme is still secure under the LRSW assumption.

We construct a SeqAS scheme based on the modified CL signature scheme that supports "public key sharing" by using the "randomness re-use" technique of Lu et al. [28]. It is easy to sequentially aggregate signatures if the element $Y$ is shared with all signers since we only need to consider the aggregation of the $\left\{X_{i}\right\}$ values of signers instead of the $\left\{X_{i}, Y_{i}\right\}$ values of signers. For instance, the first signer who has a private key $x_{1}$ generates a signature $\sigma_{1}=\left(A_{1}, B_{1}, C_{1}\right)$ on a message $M_{1}$ as $A_{1}=g^{r_{1}}, B_{1}=Y^{r_{1}}$, and $C_{1}=$ $\left(g^{r_{1}}\right)^{x_{1}}\left(Y^{r_{1}}\right)^{x_{1} M_{1}}$. The second signer with a private key $x_{2}$ generates a sequential aggregate signature $\sigma_{2}=$ $\left(A_{2}, B_{2}, C_{2}\right)$ on a message $M_{2}$ as $A_{2}=A_{1}, B_{2}=B_{1}$, and $C_{2}=C_{1}\left(A_{1}\right)^{x_{2}}\left(B_{1}\right)^{x_{2} M_{2}}$ by using the "randomness re-use" technique. Therefore a sequential aggregate signature of signers is formed as $\sigma_{\Sigma}=\left(A=g^{r}, B=\right.$ $\left.Y^{r}, C=A^{\sum x_{i}} B^{\sum x_{i} M_{i}}\right)$. Additionally, each signer should re-randomize the aggregate signature to prevent a simple attack.

\subsection{Construction}

Our SeqAS scheme is described as follows: 
SeqAS.Setup $\left(1^{\lambda}\right)$ : This algorithm first generates the bilinear groups $\mathbb{G}, \mathbb{G}_{T}$ of prime order $p$ of bit size $\Theta(\lambda)$. Let $g$ be the generator of $\mathbb{G}$. It chooses a random element $Y \in \mathbb{G}$ and outputs public parameters as $P P=\left(p, \mathbb{G}, \mathbb{G}_{T}, e, g, Y\right)$.

SeqAS.KeyGen $(P P)$ : This algorithm takes as input the public parameters $P P$. It selects a random exponent $x \in \mathbb{Z}_{p}$ and sets $X=g^{x}$. Then it outputs a private key as $S K=x$ and a public key as $P K=X$.

SeqAS.AggSign $\left(\sigma_{\Sigma}^{\prime}, \mathbf{M}^{\prime}, \mathbf{P K} \mathbf{K}^{\prime}, M, S K, P P\right)$ : This algorithm takes as input an aggregate-so-far $\sigma_{\Sigma}^{\prime}=\left(A^{\prime}, B^{\prime}, C^{\prime}\right)$ on messages $\mathbf{M}^{\prime}=\left(M_{1}, \ldots, M_{k}\right)$ under public keys $\mathbf{P} \mathbf{K}^{\prime}=\left(P K_{1}, \ldots, P K_{k}\right)$ where $P K_{i}=X_{i}$, a message $M \in \mathbb{Z}_{p}^{*}$, and a private key $S K=x$ with $P P$. It first checks the validity of $\sigma_{\Sigma}^{\prime}$ by calling $\operatorname{AggVerify}\left(\sigma_{\Sigma}^{\prime}, \mathbf{M}^{\prime}, \mathbf{P K}, P P\right)$. If $\sigma_{\Sigma}^{\prime}$ is not valid, then it halts. It checks that the public key $P K$ of $S K$ does not already exist in $\mathbf{P} \mathbf{K}^{\prime}$. If the public key already exists, then it halts. Note that if $k=0$, then $\sigma_{\Sigma}^{\prime}=(g, Y, 1)$. It selects a random exponent $r \in \mathbb{Z}_{p}$ and computes

$$
A=\left(A^{\prime}\right)^{r}, B=\left(B^{\prime}\right)^{r}, C=\left(C^{\prime} \cdot\left(A^{\prime}\right)^{x} \cdot\left(B^{\prime}\right)^{x M}\right)^{r} .
$$

It outputs an aggregate signature as $\sigma_{\Sigma}=(A, B, C)$.

SeqAS.AggVerify $\left(\sigma_{\Sigma}, \mathbf{M}, \mathbf{P K}, P P\right)$ : This algorithm takes as input an aggregate signature $\sigma_{\Sigma}=(A, B, C)$ on messages $\mathbf{M}=\left(M_{1}, \ldots, M_{l}\right)$ under public keys $\mathbf{P K}=\left(P K_{1}, \ldots, P K_{l}\right)$ where $P K_{i}=X_{i}$. It first checks that any $M_{i}$ is in $\mathbb{Z}_{p}^{*}$, any public key does not appear twice in $\mathbf{P K}$, and any public key in $\mathbf{P K}$ has been certified. If these checks fail, then it outputs 0 . If $l=0$, then it outputs 1 if $\sigma_{\Sigma}=(1, Y, 1), 0$ otherwise. Next, it verifies that

$$
e(A, Y) \stackrel{?}{=} e(B, g) \text { and } e(C, g) \stackrel{?}{=} e\left(A, \prod_{i=1}^{l} X_{i}\right) \cdot e\left(B, \prod_{i=1}^{l} X_{i}^{M_{i}}\right)
$$

If these equations hold, then it outputs 1 . Otherwise, it outputs 0.

A sequential aggregate signature $\sigma_{\Sigma}=(A, B, C)$ on messages $\mathbf{M}=\left(M_{1}, \ldots, M_{l}\right)$ under public keys $\mathbf{P K}=$ $\left(P K_{1}, \ldots, P K_{l}\right)$ has the following form

$$
A=g^{r}, B=Y^{r}, C=\left(g^{r}\right)^{\sum_{i=1}^{l} x_{i}}\left(Y^{r}\right)^{\sum_{i=1}^{l} x_{i} M_{i}}
$$

where $P K_{i}=X_{i}=g^{x_{i}}$.

\subsection{Security Analysis}

We prove the security of our SeqAS scheme based on the security of the CL signature scheme without random oracles. Therefore, our SeqAS scheme is existentially unforgeable under a chosen message attack under the LRSW assumption since the security of the CL signature scheme is proven under the LRSW assumption.

Theorem 3.3. The above SeqAS scheme is existentially unforgeable under a chosen message attack if the $C L$ signature scheme is existentially unforgeable under a chosen message attack. That is, for any PPT adversary $\mathcal{A}$ for the above SeqAS scheme, there exists a PPT algorithm $\mathcal{B}$ for the $C L$ signature scheme such that $\boldsymbol{A} \boldsymbol{d} \boldsymbol{v}_{\mathcal{A}}^{\operatorname{SeqAS}}(\boldsymbol{\lambda}) \leq \boldsymbol{A} \boldsymbol{d} \boldsymbol{v}_{\mathcal{B}}^{C L}(\lambda)$. 
Proof. The main idea of the security proof is that the aggregated signature of our SeqAS scheme is independent of the order of aggregation, and the simulator of the SeqAS scheme possesses the private keys of all signers except the private key of the challenge public key. That is, if the adversary requests a sequential aggregate signature, then the simulator first obtains a CL signature from the target scheme's signing oracle and runs the aggregate signing algorithm to generate a sequential aggregate signature. If the adversary finally outputs a forged sequential aggregate signature that is non-trivial, then the simulator extracts the CL signature of the challenge public key from the forged aggregate signature by using the private keys of other signers.

Suppose there exists an adversary $\mathcal{A}$ that forges the above SeqAS scheme with non-negligible advantage $\varepsilon$. A simulator $\mathcal{B}$ that forges the $C L$ signature scheme is first given: a challenge public key $P K_{C L}=\left(p, \mathbb{G}, \mathbb{G}_{T}, e, g, X, Y\right)$. Then $\mathcal{B}$ that interacts with $\mathcal{A}$ is described as follows:

Setup: $\mathcal{B}$ first constructs $P P=\left(p, \mathbb{G}, \mathbb{G}_{T}, e, g, Y\right)$ and $P K^{*}=X$ from $P K_{C L}$. Next, it initializes a key-pair list KeyList as an empty one and gives $P P$ and $P K^{*}$ to $\mathcal{A}$.

Certification Query: $\mathcal{A}$ adaptively requests the certification of a public key by providing a public key $P K_{i}=X_{i}$ and its private key $S K_{i}=x_{i}$. $\mathcal{B}$ checks the private key and adds the key pair $\left(P K_{i}, S K_{i}\right)$ to KeyList.

Signature Query: $\mathcal{A}$ adaptively requests a sequential aggregate signature by providing an aggregate-so-far $\sigma_{\Sigma}^{\prime}$ on messages $\mathbf{M}^{\prime}=\left(M_{1}, \ldots, M_{k}\right)$ under public keys $\mathbf{P} \mathbf{K}^{\prime}=\left(P K_{1}, \ldots, P K_{k}\right)$, and a message $M$ to sign under the challenge private key of $P K^{*} . \mathcal{B}$ proceeds the aggregate signature query as follows:

1. It first checks that the signature $\sigma_{\Sigma}^{\prime}$ is valid by calling SeqAS.AggVerify and that each public key in PK' exits in KeyList.

2. It queries its signing oracle that simulates PKS.Sign on the message $M$ for the challenge public key $P K^{*}$ and obtains a signature $\sigma$.

3. For each $1 \leq i \leq k$, it constructs an aggregate signature on message $M_{i}$ using SeqAS.AggSign since it knows the private key that corresponds to $P K_{i}$. The resulting signature is an aggregate signature for messages $\mathbf{M}^{\prime}|| M$ under public keys $\mathbf{P} \mathbf{K}^{\prime}|| P K^{*}$ since this scheme does not check the order of aggregation. It gives the result signature $\sigma_{\Sigma}$ to $\mathcal{A}$.

Output: $\mathcal{A}$ outputs a forged aggregate signature $\sigma_{\Sigma}^{*}=\left(A^{*}, B^{*}, C^{*}\right)$ on messages $\mathbf{M}^{*}=\left(M_{1}, \ldots, M_{l}\right)$ under public keys $\mathbf{P K}^{*}=\left(P K_{1}, \ldots, P K_{l}\right)$ for some $l$. Without loss of generality, we assume that $P K_{1}=P K^{*}$. $\mathcal{B}$ proceeds as follows:

1. It first checks the validity of $\sigma_{\Sigma}^{*}$ by calling SeqAS.AggVerify. Additionally, the forged signature should not be trivial: the challenge public key $P K^{*}$ must be in $\mathbf{P K} \mathbf{K}^{*}$, and the message $M_{1}$ must not be queried by $\mathcal{A}$ to the signature query oracle.

2. For each $2 \leq i \leq l$, it parses $P K_{i}=X_{i}$ from $\mathbf{P K} \mathbf{K}^{*}$, and it retrieves the private key $S K_{i}=x_{i}$ of $P K_{i}$ from KeyList. It then computes

$$
A=A^{*}, B=B^{*}, C=C^{*} \cdot\left(\left(A^{*}\right)^{\sum_{i=2}^{l} x_{i}}\left(B^{*}\right)^{\sum_{i=2}^{l} x_{i} M_{i}}\right)^{-1} .
$$

3. It outputs $\sigma^{*}=(A, B, C)$ on a message $M^{*}=M_{1}$ as a non-trivial forgery of the CL signature scheme since it did not make a signing query on $M_{1}$. 
To finish the proof, we first show that the distribution of the simulation is correct. It is obvious that the public parameters and the public key are correctly distributed. The distribution of the sequential aggregate signatures is correct since this scheme does not check the order of aggregation. Finally, we can show that the resulting signature $\sigma^{*}=(A, B, C)$ of the simulator is a valid signature for the CL signature scheme on the message $M_{1}$ under the public key $P K^{*}$ since it satisfies the following equation:

$$
\begin{aligned}
e(C, g) & =e\left(C^{*} \cdot\left(\left(A^{*}\right)^{\sum_{i=2}^{l} x_{i}}\left(B^{*}\right)^{\sum_{i=2}^{l} x_{i} M_{i}}\right)^{-1}, g\right) \\
& =e\left(\left(A^{*}\right)^{\sum_{i=1}^{l} x_{i}}\left(B^{*}\right)^{\sum_{i=1}^{l} x_{i} M_{i}} \cdot\left(A^{*}\right)^{-\sum_{i=2}^{l} x_{i}}\left(B^{*}\right)^{-\sum_{i=2}^{l} x_{i} M_{i}}, g\right) \\
& =e\left(\left(A^{*}\right)^{x_{1}}\left(B^{*}\right)^{x_{1} M_{1}}, g\right)=e\left(A^{*}, g^{x_{1}}\right) \cdot e\left(B^{*}, g^{x_{1} M_{1}}\right) \\
& =e(A, X) \cdot e\left(B, X^{M^{*}}\right) .
\end{aligned}
$$

This completes our proof.

\subsection{Discussions}

Efficiency. The public key of our SeqAS scheme consists of just one group element and the aggregate signature consists of three group elements, since the public key element $Y$ of the CL signature scheme is moved to the public parameters of our scheme. The aggregate signing algorithm requires one aggregate verification and five exponentiations, and the aggregate verification algorithm requires five pairing operations and $l$ exponentiations where $l$ is the number of signers in the aggregate signature. In the SeqAS scheme of Schröder [33], the public key consists of two group elements, the aggregate signature consists of four group elements, and the aggregate verification algorithm requires $l$ pairing operations and $l$ exponentiations. Therefore, our SeqAS scheme is more efficient than the SeqAS scheme of Schröder.

Asymmetric Bilinear Groups. We can use asymmetric bilinear groups instead of symmetric bilinear groups to reduce the size of aggregate signatures. Let $\mathbb{G}, \widehat{\mathbb{G}}, \mathbb{G}_{T}$ be the cyclic groups of prime order $p$. We say that $\mathbb{G}, \hat{\mathbb{G}}, \mathbb{G}_{T}$ are asymmetric bilinear groups if there exists the bilinear map $e: \mathbb{G} \times \widehat{\mathbb{G}} \rightarrow \mathbb{G}_{T}$ that has bilinearity and non-degeneracy properties. The SeqAS scheme in asymmetric bilinear groups is described as follows: the public parameters is $P P=\left(p, \mathbb{G}, \hat{\mathbb{G}}, \mathbb{G}_{T}, e, g, \hat{g}, Y, \hat{Y}\right)$, the private key and the public key are $S K=x$ and $P K=\hat{X} \in \hat{\mathbb{G}}$ respectively, and the aggregate signature is $\sigma_{\Sigma}=\left(A=g^{r}, B=Y^{r}, C=A^{\sum x_{i}} B^{\sum x_{i} M_{i}}\right) \in \mathbb{G}^{3}$. For instance, if we instantiate the asymmetric bilinear groups using the 175-bit MNT curve with embedding degree 6 to guarantee 80-bit security level, the size of public key is 525 bit and the size of aggregate signature is 525 bit. In the 175-bit MNT curve, the SeqAS scheme of Lu et al. [28] has 113 kilo-bits size of the public key and 350 bit size of the aggregate signature, and the SeqAS scheme of Schröder [33] has 1050 bit size of the public key and 700 bit size of the aggregate signature.

Public-Key Signature. We can easily derive a new PKS scheme from our SeqAS scheme. Compared to the CL signature scheme, the new PKS scheme has an advantage such that the public key just consists of one group element $X$ instead of two group elements $X, Y$ since the element $Y$ is moved to the public parameters. A signer who has a private key $x$ can generate a signature $\sigma=(A, B, C)$ as $A=g^{r}, B=Y^{r}$, and $C=A^{x} B^{x M}$. A verifier can verify the signature by checking that $e(A, Y)=e(B, g)$ and $e(C, g)=e(A, X) \cdot e\left(B, X^{M}\right)$. This new PKS scheme is also secure under the LRSW assumption.

Secure Public Parameters. The public parameters of our SeqAS scheme should be generated by a trusted party to assure that the discrete logarithm of the element $Y$ is unknown. The reason is that if a party knows the value $y$ of $Y=g^{y}$ in the public parameters, then he can easily creates a signature for any signer as $\sigma=\left(A=g^{r}, B=Y^{r}, C=X^{r} X^{r y M}\right)$ where $X$ is the public key of the signer. To remove the trust assumption 
in the setup algorithm, we may use a hash function $H:\{0,1\}^{*} \rightarrow \mathbb{G}$. That is, any party can generate public parameters $P P$ such that $Y=H(L)$ where $L$ is a fixed label string. In this case, the party cannot know the discrete logarithm of $Y$ because of the property of the hash function. Note that this hash function can be implemented by using an admissible encoding function [12].

\section{Synchronized Aggregate Signature}

In this section, we first define the synchronized aggregate signature and its security model. Next, we propose an efficient synchronized aggregate signature scheme based on the CL signature scheme, and prove its security in the random oracle model under the LRSW assumption.

\subsection{Definitions}

Synchronized aggregate signature (SyncAS) is a special type of public-key aggregate signature (PKAS) that allows anyone to aggregate signer's signatures on different messages with a same time period into a short aggregate signature if all signers have the synchronized time period information like a clock [1, 22]. In SyncAS scheme, each signer has a synchronized time period or has an access to public time information. Each signer can generate an individual signature on a message $M$ and a time period $w$. Note that the signer can generate just one signature per one time period. After that, anyone can aggregate individual signatures of other signers into a short aggregate signature $\sigma_{\Sigma}$ if the individual signatures are generated on the same time period $w$. The resulting aggregate signature has the same size of the individual signature.

Definition 4.1 (Synchronized Aggregate Signature). A synchronized aggregate signature (SyncAS) scheme consists of six PPT algorithms Setup, KeyGen, Sign, Verify, Aggregate, and AggVerify, which are defined as follows:

$\operatorname{Setup}\left(1^{\lambda}\right)$. The setup algorithm takes as input a security parameter $1^{\lambda}$ and outputs public parameters PP.

$\operatorname{KeyGen}(P P)$. The key generation algorithm takes as input the public parameters $P P$, and outputs a public key $P K$ and a private key $S K$.

$\operatorname{Sign}(M, w, S K, P P)$. The signing algorithm takes as input a message $M$, a time period $w$, and a private key $S K$ with $P P$, and outputs an individual signature $\sigma$.

$\operatorname{Verify}(\sigma, M, P K, P P)$. The verification algorithm takes as input a signature $\sigma$ on a message $M$ under a public key $P K$, and outputs either 1 or 0 depending on the validity of the signature.

Aggregate $(\mathbf{S}, \mathbf{M}, \mathbf{P K})$. The aggregation algorithm takes as input individual signatures $\mathbf{S}=\left(\sigma_{1}, \ldots, \sigma_{l}\right)$ on messages $\mathbf{M}=\left(M_{1}, \ldots, M_{l}\right)$ under public keys $\mathbf{P K}=\left(P K_{1}, \ldots, P K_{l}\right)$, and outputs an aggregate signature $\sigma_{\Sigma}$.

$\operatorname{AggVerify}\left(\sigma_{\Sigma}, \mathbf{M}, \mathbf{P K}, P P\right)$. The aggregate verification algorithm takes as input an aggregate signature $\sigma_{\Sigma}$ on messages $\mathbf{M}=\left(M_{1}, \ldots, M_{l}\right)$ under public keys $\mathbf{P K}=\left(P K_{1}, \ldots, P K_{l}\right)$, and outputs either 1 or 0 depending on the validity of the aggregate signature.

The correctness requirement is that for each PP output by Setup, for all $(P K, S K)$ output by KeyGen, any $M$, we have that AggVerify $($ Aggregate $(\mathbf{S}, \mathbf{M}, \mathbf{P K}), \mathbf{M}, \mathbf{P K}, P P)=1$ where $\mathbf{S}$ is individual signatures on messages $\mathbf{M}$ under public keys $\mathbf{P K}$. 
The security model of SyncAS was introduced by Gentry and Ramzan [22]. In this paper, we follow the security model that was proposed by Ahn et al. [1]. The security model of Ahn et al. is a more restricted model that requires the adversary to correctly generate other signers' public keys and private keys except the challenge signer's key. To ensure the correct generation of public keys and private keys, the adversary should submit the private key of the public key, or he should prove that he knows the corresponding private key by using zero-knowledge proofs.

In the security model of SyncAS, the public parameters and the challenge public key $P K^{*}$ are given to the adversary at first. The adversary can request the certification of a public key through a certification query by providing the public key and the corresponding private key. It also can request a signature on a message $M$ and a time period $w$ that was not used before under the challenge public key. Finally, it outputs a forged synchronized aggregate signature $\sigma_{\Sigma}^{*}$. The adversary breaks the SyncAS scheme if the forged synchronized aggregate signature of the adversary is valid and non-trivial.

Definition 4.2 (Unforgeability). The security notion of existential unforgeability under a chosen message attack is defined in terms of the following experiment between a challenger $\mathcal{C}$ and a PPT adversary $\mathcal{A}$ :

1. Setup: $\mathcal{C}$ first initializes a key-pair list KeyList as empty. Next, it runs Setup to obtain public parameters $P P$ and KeyGen to obtain a key pair $(P K, S K)$, and gives $P K$ to $\mathcal{A}$.

2. Certification Query: $\mathcal{A}$ adaptively requests the certification of a public key by providing a key pair $(P K, S K)$. Then $\mathcal{C}$ adds the key pair $(P K, S K)$ to KeyList if the key pair is a valid one.

3. Hash Query: $\mathcal{A}$ adaptively requests a hash on a string for various hash functions, and receives a hash value.

4. Signature Query: $\mathcal{A}$ adaptively requests a signature on a message $M$ and a time period $w$ that was not used before to sign under the challenge public key $P K$, and receives an individual signature $\sigma$.

5. Output: Finally (after a sequence of the above queries), $\mathcal{A}$ outputs a forged synchronized aggregate signature $\sigma_{\Sigma}^{*}$ on messages $\mathbf{M}^{*}$ under public keys $\mathbf{P} \mathbf{K}^{*} . \mathcal{C}$ outputs 1 if the forged signature satisfies the following three conditions, or outputs 0 otherwise: 1) $\left.\mathbf{A g g V e r i f y}\left(\sigma_{\Sigma}^{*}, \mathbf{M}^{*}, \mathbf{P K} \mathbf{K}^{*}, P P\right)=1,2\right)$ The challenge public key PK must exist in $\mathbf{P} \mathbf{K}^{*}$ and each public key in $\mathbf{P} \mathbf{K}^{*}$ except the challenge public key must be in KeyList, and 3) The corresponding message $M$ in $\mathbf{M}^{*}$ of the challenge public key PK must not have been queried by $\mathcal{A}$ to the signing oracle.

The advantage of $\mathcal{A}$ is defined as $\boldsymbol{A d} \boldsymbol{v}_{\mathcal{A}}^{\text {SyncAS }}=\operatorname{Pr}[\mathcal{C}=1]$ where the probability is taken over all the randomness of the experiment. A SyncAS scheme is existentially unforgeable under a chosen message attack if all PPT adversaries have at most a negligible advantage (for large enough security parameter) in the above experiment.

\subsection{Design Principle}

We first describe the design idea of our SyncAS scheme. In the previous section, we proposed a modified $\mathrm{CL}$ signature scheme that shares the element $Y$ in the public parameters. The signature of this modified $\mathrm{CL}$ signature scheme is formed as $\sigma=\left(A=g^{r}, B=Y^{r}, C=A^{x} B^{x M}\right)$. If we can force signers to use the same $A=g^{r}$ and $B=Y^{r}$ in signatures, then we easily obtain an aggregate signature as $\sigma_{\Sigma}=\left(A=g^{r}, B=Y^{r}, C=\right.$ $A^{\sum x_{i}} B^{\sum x_{i} M_{i}}$ by just multiplying individual signatures of signers. In synchronized aggregate signatures, it is possible to force signers to use the same $A$ and $B$ since all signers have the same time period $w$. Therefore, 
each signer first sets $A=H(0 \| w)$ and $B=H(1 \| w)$ using the hash function $H$ and the time period $w$, and then he generates an individual signature $\sigma=\left(C=A^{x} B^{x M}, w\right)$. We need to hash a message for the proof of security.

\subsection{Construction}

Let $\mathcal{W}$ be a set of time periods where $|\mathcal{W}|$ is fixed polynomial in the security parameter 1 . Our SyncAS scheme is described as follows:

SyncAS.Setup $\left(1^{\lambda}\right)$ : This algorithm first generates the bilinear groups $\mathbb{G}, \mathbb{G}_{T}$ of prime order $p$ of bit size $\Theta(\lambda)$. Let $g$ be the generator of $\mathbb{G}$. It chooses two hash functions $H_{1}:\{0,1\} \times \mathcal{W} \rightarrow \mathbb{G}$ and $H_{2}$ : $\{0,1\}^{*} \times \mathcal{W} \rightarrow \mathbb{Z}_{p}^{*}$. It outputs public parameters as $P P=\left(p, \mathbb{G}, \mathbb{G}_{T}, e, g, H_{1}, H_{2}\right)$.

SyncAS.KeyGen $(P P)$ : This algorithm takes as input the public parameters $P P$. It selects a random exponent $x \in \mathbb{Z}_{p}$ and sets $X=g^{x}$. Then it outputs a private key as $S K=x$ and a public key as $P K=X$.

$\operatorname{SyncAS.Sign}(M, w, S K, P P):$ This algorithm takes as input a message $M \in\{0,1\}^{*}$, a time period $w \in \mathcal{W}$, and a private key $S K=x$ with $P P$. It first sets $A=H_{1}(0|| w), B=H_{1}(1|| w), h=H_{2}(M \| w)$ and computes $C=A^{x} B^{x h}$. It outputs a signature as $\sigma=(C, w)$.

SyncAS.Verify $(\sigma, M, P K, P P)$ : This algorithm takes as input a signature $\sigma=(C, w)$ on a message $M$ under a public key $P K=X$. It first checks that the public key has been certified. If these checks fail, then it outputs 0 . Next, it sets $A=H_{1}(0 \| \mid w), B=H_{1}(1|| w), h=H_{2}(M \| w)$ and verifies that $e(C, g) \stackrel{?}{=}$ $e\left(A B^{h}, X\right)$. If this equation holds, then it outputs 1 . Otherwise, it outputs 0 .

SyncAS.Aggregate( $\mathbf{S}, \mathbf{M}, \mathbf{P K}, P P)$ : This algorithm takes as input signatures $\mathbf{S}=\left(\sigma_{1}, \ldots, \sigma_{l}\right)$ on messages $\mathbf{M}=\left(M_{1}, \ldots, M_{l}\right)$ under public keys $\mathbf{P K}=\left(P K_{1}, \ldots, P K_{l}\right)$ where $\sigma_{i}=\left(C_{i}^{\prime}, w_{i}^{\prime}\right)$ and $P K_{i}=X_{i}$. It first checks that that $w_{1}^{\prime}$ is equal to $w_{i}^{\prime}$ for $i=2$ to $l$. If it fails, it halts. Next, it sets $w=w_{1}^{\prime}$ and computes $C=\prod_{i=1}^{l} C_{i}^{\prime}$. It outputs an aggregate signature as $\sigma_{\Sigma}=(C, w)$.

SyncAS.AggVerify $\left(\sigma_{\Sigma}, \mathbf{M}, \mathbf{P K}, P P\right)$ : This algorithm takes as input an aggregate signature $\sigma_{\Sigma}=(C, w)$ on messages $\mathbf{M}=\left(M_{1}, \ldots, M_{l}\right)$ under public keys $\mathbf{P K}=\left(P K_{1}, \ldots, P K_{l}\right)$ where $P K_{i}=X_{i}$. It first checks that any public key does not appear twice in $\mathbf{P K}$ and any public key in $\mathbf{P K}$ has been certified. If these checks fail, then it outputs 0 . Next, it sets $A=H_{1}(0 \| w), B=H_{1}(1|| w), h_{i}=H_{2}\left(M_{i} \| w\right)$ for all $1 \leq i \leq l$ and verifies that

$$
e(C, g) \stackrel{?}{=} e\left(A, \prod_{i=1}^{l} X_{i}\right) \cdot e\left(B, \prod_{i=1}^{l} X_{i}^{h_{i}}\right) .
$$

If this equation holds, then it outputs 1 . Otherwise, it outputs 0.

A synchronized aggregate signature $\sigma_{\Sigma}=(C, w)$ on messages $\mathbf{M}=\left(M_{1}, \ldots, M_{l}\right)$ under public keys $\mathbf{P K}=$ $\left(P K_{1}, \ldots, P K_{l}\right)$ has the following form

$$
C=H_{1}(0 \| w)^{\sum_{i=1}^{l} x_{i}} H_{1}(1|| w)^{\sum_{i=1}^{l} x_{i} H_{2}\left(M_{i} \| w\right)}
$$

where $P K_{i}=X_{i}=g^{x_{i}}$.

\footnotetext{
${ }^{1}$ The set $\mathcal{W}$ does not need to be included in $P P$ since an integer $w$ in the range $[1, T]$ can be used where $T$ is fixed polynomial in the security parameter. In practice, we can set $T=2^{32}$ if the maximum time period of certificates is 10 years and a signer generates a signature per each second. The previous SyncAS schemes [1, 22] support exponential size of time periods while our SyncAS scheme supports polynomial size of time periods.
} 


\subsection{Security Analysis}

We first prove the security of our SyncAS scheme based on the security of the CL signature scheme in the random oracle model. Next, we show that our SyncAS scheme can be proven to be secure under the OT-LRSW assumption.

Theorem 4.3. The above SyncAS scheme is existentially unforgeable under a chosen message attack if the $C L$ signature scheme is existentially unforgeable under a chosen message attack. That is, for any PPT adversary $\mathcal{A}$ for the above SyncAS scheme, there exists a PPT algorithm $\mathcal{B}$ for the $C L$ signature scheme such that $\boldsymbol{A d} \boldsymbol{v}_{\mathcal{A}}^{\text {SyncAS }}(\lambda) \leq|\mathcal{W}| \cdot q_{H_{2}} \cdot \boldsymbol{A d} \boldsymbol{d}_{\mathcal{B}}^{C L}(\lambda)$ where $q_{H_{2}}$ is the maximum number of $H_{2}$ hash queries.

Proof. The main idea of the security proof is that the random oracle model supports the programmability of hash functions, the adversary can request just one signature per one time period in this security model, and the simulator possesses the private keys of all signers except the private key of the challenge public key. In the proof, the simulator first guesses the time period $w^{\prime}$ of the forged synchronized aggregate signature and selects a random query index $k$ of the hash function $H_{2}$. After that, if the adversary requests a signature on a message $M$ and a time period $w$ such that $w \neq w^{\prime}$, then he can easily generate the signature by using the programmability of the random oracle model. If the adversary requests a signature for the time period $w=w^{\prime}$, then he can generate the signature if the query index $i$ is equal to the index $k$. Otherwise, the simulator should abort the simulation. Finally, if the adversary outputs a forged synchronized aggregate signature that is non-trivial on the time period $w^{\prime}$, then the simulator extracts the CL signature of the challenge public key from the forged aggregate signature by using the private keys of other signers.

Suppose there exists an adversary $\mathcal{A}$ that forges the above SyncAS scheme with non-negligible advantage $\varepsilon$. A simulator $\mathcal{B}$ that forges the CL signature scheme is first given: a challenge public key $P K_{C L}=\left(p, \mathbb{G}, \mathbb{G}_{T}, e, g, X, Y\right)$. Then $\mathcal{B}$ that interacts with $\mathcal{A}$ is described as follows:

Setup: $\mathcal{B}$ first constructs $P P=\left(p, \mathbb{G}, \mathbb{G}_{T}, e, g, H_{1}, H_{2}\right)$ and $P K^{*}=X$ from $P K_{C L}$. It chooses a random value $h^{\prime} \in \mathbb{Z}_{p}^{*}$ and queries its signing oracle PKS.Sign to obtain $\sigma^{\prime}=\left(A^{\prime}, B^{\prime}, C^{\prime}\right)$. Let $q_{H_{1}}$ and $q_{H_{2}}$ be the maximum number of $H_{1}$ and $H_{2}$ hash queries respectively. It chooses a random index $k$ such that $1 \leq k \leq q_{H_{2}}$ and guesses a random time period $w^{\prime} \in \mathcal{W}$ of the forged signature. Next, it initializes a key-pair list KeyList, hash lists $H_{1}$-List,$H_{2}$-List as an empty one and gives $P P$ and $P K^{*}$ to $\mathcal{A}$.

Certification Query: $\mathcal{A}$ adaptively requests the certification of a public key by providing a public key $P K_{i}=X_{i}$ and its private key $S K_{i}=x_{i}$. $\mathcal{B}$ checks the private key and adds the key-pair $\left(P K_{i}, S K_{i}\right)$ to KeyList.

Hash Query: $\mathcal{A}$ adaptively requests a hash value for $H_{1}$ and $H_{2}$ respectively. If this is a $H_{1}$ hash query on a bit $b \in\{0,1\}$ and a time period $w_{i}$, then $\mathcal{B}$ treats the query as follows:

- If $b=0$ and $w_{i} \neq w^{\prime}$, then it selects a random exponent $r_{0, i} \in \mathbb{Z}_{p}$ and sets $H_{1}\left(0 \| w_{i}\right)=g^{r_{0, i}}$.

- If $b=0$ and $w_{i}=w^{\prime}$, then it sets $H_{1}\left(0|| w_{i}\right)=A^{\prime}$.

- If $b=1$ and $w_{i} \neq w^{\prime}$, then it selects a random exponent $r_{1, i} \in \mathbb{Z}_{p}$ and sets $H_{1}\left(1 \| w_{i}\right)=g^{r_{1, i}}$.

- If $b=1$ and $w_{i}=w^{\prime}$, then it sets $H_{1}\left(1|| w_{i}\right)=B^{\prime}$.

If this is a $H_{2}$ hash query on a message $M_{i}$ and a time period $w_{j}$, then $\mathcal{B}$ treats the query as follows:

- If $i \neq k$ or $w_{j} \neq w^{\prime}$, then it selects a random value $h_{i, j} \in \mathbb{Z}_{p}$ and sets $H_{2}\left(M_{i} \| w_{j}\right)=h_{i, j}$.

- If $i=k$ and $w_{j}=w^{\prime}$, then it sets $H_{2}\left(M_{i}|| w_{j}\right)=h^{\prime}$. 
Note that $\mathcal{B}$ keeps the tuple $\left(b, w_{i}, r_{b, i}, H_{1}\left(b \| w_{i}\right)\right)$ in $H_{1}$-List and the tuple $\left(M_{i}, w_{j}, h_{i, j}\right)$ in $H_{2}-L i s t$.

Signature Query: $\mathcal{A}$ adaptively requests a signature by providing a message $M_{i}$ and a time period $w_{j}$ to sign under the challenge private key of $P K^{*} . \mathcal{B}$ proceeds the signature query as follows:

- If $w_{i} \neq w^{\prime}$, then it responds $\sigma_{i, j}=\left(X^{r_{0, i}} X^{r_{1, i}} h_{i, j}, w_{j}\right)$ where $r_{0, i}, r_{1, i}$, and $h_{i, j}$ are retrieved from the $H_{1}$-List and $H_{2}$-List.

- If $w_{i}=w^{\prime}$ and $i=k$, then it responds $\sigma_{i, j}=\left(C^{\prime}, w_{j}\right)$.

- If $w_{i}=w^{\prime}$ and $i \neq k$, it aborts the simulation.

Output: $\mathcal{A}$ outputs a forged aggregate signature $\sigma_{\Sigma}^{*}=\left(C^{*}, w^{*}\right)$ on messages $\mathbf{M}^{*}=\left(M_{1}, \ldots, M_{l}\right)$ under public keys $\mathbf{P K} \mathbf{K}^{*}=\left(P K_{1}, \ldots, P K_{l}\right)$ for some $l$. Without loss of generality, we assume that $P K_{1}=P K^{*}$. B proceeds as follows:

1. It checks the validity of $\sigma_{\Sigma}^{*}$ by calling SyncAS.AggVerify. Additionally, the forged signature should not be trivial: the challenge public key $P K^{*}$ must be in $\mathbf{P K}{ }^{*}$, and the message $M_{1}$ must not be queried by $\mathcal{A}$ to the signature query oracle.

2. If $w^{*} \neq w^{\prime}$, then it aborts the simulation since it fails to guess the forged time period.

3. For each $2 \leq i \leq l$, it retrieves the private key $S K_{i}=x_{i}$ of $P K_{i}$ from KeyList and sets $h_{i, *}=H_{2}\left(M_{i} \| w^{*}\right)$. Next, it computes

$$
A=A^{\prime}, B=B^{\prime}, C=C^{*} \cdot\left(\left(A^{\prime}\right)^{\sum_{i=2}^{l} x_{i}}\left(B^{\prime}\right)^{\sum_{i=2}^{l} x_{i} h_{i, *}}\right)^{-1}
$$

4. If $H_{2}\left(M_{1} \| w^{*}\right)=h^{\prime}$, then it also aborts the simulation.

5. It outputs $\sigma^{*}=(A, B, C)$ on a message $h_{1, *}$ as a non-trivial forgery of the CL signature scheme since $h_{1, *} \neq h^{\prime}$ where $h_{1, *}=H_{2}\left(M_{1} \| w^{*}\right)$.

To finish the proof, we first show that the distribution of the simulation is correct. It is obvious that the public parameters and the public key are correctly distributed. The distribution of the signatures is also correct. Next, we show that the resulting signature $\sigma^{*}=(A, B, C)$ of the simulator is a valid signature for the CL signature scheme on the message $h_{1, *} \neq h^{\prime}$ under the public key $P K^{*}$ since it satisfies the following equation:

$$
\begin{aligned}
e(C, g) & =e\left(C^{*} \cdot\left(\left(A^{\prime}\right)^{\sum_{i=2}^{l} x_{i}}\left(B^{\prime}\right)^{\sum_{i=2}^{l} x_{i} H_{2}\left(M_{i} \| w^{*}\right)}\right)^{-1}, g\right) \\
& =e\left(\left(A^{\prime}\right)^{\sum_{i=1}^{l} x_{i}}\left(B^{\prime}\right)^{\sum_{i=1}^{l} x_{i} h_{i, *}} \cdot\left(A^{\prime}\right)^{-\sum_{i=2}^{l} x_{i}}\left(B^{\prime}\right)^{-\sum_{i=2}^{l} x_{i} h_{i, *}}, g\right) \\
& =e\left(\left(A^{\prime}\right)^{x_{1}}\left(B^{\prime}\right)^{x_{1} h_{1, *}}, g\right)=e\left(A^{\prime}, g^{x_{1}}\right) \cdot e\left(B^{\prime}, g^{x_{1} h_{1, *}}\right) \\
& =e\left(A^{\prime}, X\right) \cdot e\left(B^{\prime}, X^{h_{1, *}}\right) .
\end{aligned}
$$

We now analyze the success probability of the simulator $\mathcal{B}$. At first, $\mathcal{B}$ succeeds the simulation if he does not abort in the simulation of signature queries and he correctly guesses the time period $w^{*}$ such that $w^{*}=w^{\prime}$ in the forged aggregate signature from the adversary $\mathcal{A}$. $\mathcal{B}$ aborts the simulation of signature queries if the time period $w^{\prime}$ is given from $\mathcal{A}$ and he incorrectly guessed the index $k$ since he cannot generate a signature. Thus $\mathcal{B}$ succeeds the simulation of signature queries at least $q_{\mathrm{H}_{2}}^{-1}$ probability since the outputs 
of $\mathrm{H}_{2}$ are independently random. Next, $\mathcal{B}$ can correctly guess the time period $w^{*}$ of the forged aggregate signature with at least $|\mathcal{W}|^{-1}$ probability since he randomly chooses a random $w^{\prime}$. Note that the probability $H_{2}\left(M_{2}|| w^{*}\right)=h^{\prime}$ is negligible. Therefore, the success probability of $\mathcal{B}$ is at least $|\mathcal{W}|^{-1} \cdot q_{H_{2}}^{-1} \cdot \mathbf{A d v}_{\mathcal{A}}^{\text {SyncAS }}$ where $\mathbf{A d v}_{\mathcal{A}}^{\text {SyncAS }}$ is the success probability of $\mathcal{A}$. This completes our proof.

In the security proof, the simulator just uses a single CL signature query to answer a polynomial number of SyncAS signature queries. Thus the security of our SyncAS scheme can be proven under the OT-LRSW assumption that is weaker than the LRSW assumption. We can use this weak and static assumption to prove our SyncAS scheme since the $g^{y}$ value of a tuple $\left(A=H_{1}(0 \| w)=g^{r}, B=H_{1}(1 \| w)=g^{y r}, C=A^{x} B^{x H_{2}(M \| w)}\right)$ is not a fixed one like the LRSW assumption, but a varying one depending on a time period $w$. Thus the simulator can embed one LRSW tuple to the target time period and use the programmability of random oracles for other time periods to simulate signature queries.

Theorem 4.4. The above SyncAS scheme is existentially unforgeable under a chosen message attack if the OT-LRSW assumption holds. That is, for any PPT adversary $\mathcal{A}$ for the above SyncAS scheme, there exists a PPT algorithm $\mathcal{B}$ for the OT-LRSW assumption such that $\boldsymbol{A d v}_{\mathcal{A}}^{\text {SyncAS }}(\lambda) \leq|\mathcal{W}| \cdot q_{H_{2}} \cdot \boldsymbol{A d v}_{\mathcal{B}}^{\text {OT-LRSW }}(\lambda)$ where $q_{\mathrm{H}_{2}}$ is the maximum number of $\mathrm{H}_{2}$ hash queries.

We omit the proof of this theorem since it is almost the same with that of Theorem 4.3 .

\subsection{Discussions}

Efficiency. The public key of our SyncAS scheme consists of just one group element since our SyncAS scheme is derived from the SeqAS scheme of the previous section, and the synchronized aggregate signature consists of one group element and one integer since anyone can compute $A, B$ using the hash functions. The signing algorithm requires two group hash operations and two exponentiations, and the aggregate verification algorithm requires two group hash operations, three pairing operations, and $l$ exponentiations where $l$ is the number of signers in the aggregate signature. Our SyncAS scheme provides the shortest aggregate signature size compared to the previous SyncAS schemes [1,22] since the aggregate signature of previous SyncAS schemes consists of two group elements and one integer. Additionally the signing and verification algorithms of our scheme are efficient compared to the previous SyncAS schemes.

Asymmetric Bilinear Groups. To reduce the size of aggregate signatures, we can use asymmetric bilinear groups instead of symmetric bilinear groups. The SyncAS scheme in asymmetric bilinear groups is described as follows: the public parameters is $P P=\left(p, \mathbb{G}, \widehat{\mathbb{G}}, \mathbb{G}_{T}, e, g, \hat{g}, H_{1}, H_{2}\right)$, the private key and the public key are $S K=x$ and $P K=\hat{X}$ respectively, the individual signature is $\sigma=\left(C=H_{1}(0 \| w)^{x} H_{1}(1 \| w)^{x H_{2}(M \| w)}, w\right)$, and the aggregate signature is $\sigma_{\Sigma}=\left(C=H_{1}(0 \| w)^{\sum x_{i}} H_{1}(1|| w)^{\sum x_{i} H_{2}\left(M_{i}|| w_{i}\right)}, w\right)$. For instance, if we instantiate the asymmetric bilinear groups using the 175-bit MNT curve with embedding degree 6 to guarantee 80-bit security level, the size of aggregate signature is 207 bit since the size of a time period can be 32 bit.

Public-Key Signature. We can also derive another PKS scheme from our SyncAS scheme. The derived PKS scheme has a restriction such that a signer should use a random value $w$ that was not used before. This derived PKS scheme is the same as the CL* signature scheme that was proposed by Camenisch et al. [16]. The $\mathrm{CL}^{*}$ signature scheme supports batch verification that enables a verifier to quickly check the validity of many signatures on different messages and different signers. Compared to the CL signature scheme, the signature of the $\mathrm{CL}^{*}$ signature scheme consists of one group element and one integer instead of three group elements, and the signature verification algorithm of the $\mathrm{CL}^{*}$ signature scheme just requires two pairing operations instead of five pairing operations. 
Supporting an Exponential Size of Time Periods. In our SyncAS scheme, the size of time periods is related with the tightness of the security proof. Thus the size of time periods should be polynomial in the security parameter since the polynomial-time reduction is a standard in the security proof. However, if we consider a weaker reduction, then we can use an exponential size of time periods. In this case, we should select the large size of a group order for a secure SyncAS scheme since the success probability of a simulator is very small. For example, let the security parameter is $1^{80}$, the maximum number of hash queries of an adversary is $2^{30}$, and the size of time periods is $2^{80}$. If we want to construct a SyncAS scheme that supports 80-bits security, then we should use a CL signature scheme that supports 190-bits security. The reason is that if $\mathbf{A d v} \mathbf{v}^{C L} \leq 2^{-190}$, then we have $\mathbf{A d v} \mathbf{v}^{\text {SyncAS }} \leq 2^{-80}$ from the equation $\mathbf{A d v} \mathbf{v}^{\text {SyncAS }} \leq 2^{80} \cdot 2^{30} \cdot \mathbf{A d v} \mathbf{v}^{C L}$ of the security proof. Note that the simulator is not a polynomial-time algorithm since the success probability is very small.

Removing Random Oracles. If the number of messages is restricted to be polynomial, then Camenisch et al. [16] showed that random oracles can be removed in the CL* signature scheme by using the universal one-way hash function [31] of Canetti et al. [18,19]. We also can use the universal one-way hash function in our SyncAS scheme if the number of messages is restricted to be polynomial. However, the SeqAS scheme using the universal one-way hash function of Canetti et al. is inefficient since it requires large number of exponentiations.

\section{Combined Aggregate Signature}

In this section, we define the combined aggregate signature and propose an efficient combined aggregate signature scheme that is derived from our SeqAS scheme and our SyncAS scheme.

\subsection{Definitions}

Combined Aggregate Signature (CombAS) is a special type of PKAS that supports sequential aggregation and synchronized aggregation at the same time. Thus a CombAS scheme has the AggSign algorithm for sequential aggregation, the Sign, Verify, Aggregate algorithms for synchronized aggregation, and the AggVerify algorithm for aggregate signature verification.

Definition 5.1 (Combined Aggregate Signature). A combined aggregate signature (CombAS) scheme consists of six PPT algorithms Setup, KeyGen, Sign, Verify, AggSign, Aggregate, and AggVerify, which are defined as follows:

$\operatorname{Setup}\left(1^{\lambda}\right)$. The setup algorithm takes as input a security parameter $1^{\lambda}$ and outputs public parameters PP.

$\operatorname{KeyGen}(P P)$. The key generation algorithm takes as input the public parameters $P P$, and outputs a public key $P K$ and a private key $S K$.

$\operatorname{AggSign}\left(\sigma_{\Sigma}^{\prime}, \mathbf{M}, \mathbf{P K}, M, S K, P P\right)$. The aggregate signing algorithm takes as input an aggregate-so-far $\sigma_{\Sigma}^{\prime}$ on messages $\mathbf{M}=\left(M_{1}, \ldots, M_{k}\right)$ under public keys $\mathbf{P K}=\left(P K_{1}, \ldots, P K_{k}\right)$, a message $M$, and a private key $S K$ with $P P$, and outputs a new aggregate signature $\sigma_{\Sigma}$.

$\operatorname{Sign}(M, w, S K, P P)$. The signing algorithm takes as input a message $M$, a time period $w$, and a private key $S K$ with PP, and outputs an individual signature $\sigma$.

$\operatorname{Verify}(\sigma, M, P K, P P)$. The verification algorithm takes as input a signature $\sigma$ on a message $M$ under a public key $P K$, and outputs either 1 or 0 depending on the validity of the signature. 
Aggregate $(\mathbf{S}, \mathbf{M}, \mathbf{P K})$. The aggregation algorithm takes as input individual signatures $\mathbf{S}=\left(\sigma_{1}, \ldots, \sigma_{l}\right)$ on messages $\mathbf{M}=\left(M_{1}, \ldots, M_{l}\right)$ under public keys $\mathbf{P K}=\left(P K_{1}, \ldots, P K_{l}\right)$, and outputs an aggregate signature $\sigma_{\Sigma}$.

$\operatorname{AggVerify}\left(\sigma_{\Sigma}, \mathbf{M}, \mathbf{P K}, P P\right)$. The aggregate verification algorithm takes as input an aggregate signature $\sigma_{\Sigma}$ on messages $\mathbf{M}=\left(M_{1}, \ldots, M_{l}\right)$ under public keys $\mathbf{P K}=\left(P K_{1}, \ldots, P K_{l}\right)$, and outputs either 1 or 0 depending on the validity of the aggregate signature.

The correctness requirement is that for each PP output by Setup, for all (PK,SK) output by KeyGen, any $M$, we have that AggVerify $($ Aggregate $(\mathbf{S}, \mathbf{M}, \mathbf{P K}), \mathbf{M}, \mathbf{P K}, P P)=1$ where $\mathbf{S}$ is individual signatures on messages $\mathbf{M}$ under public keys $\mathbf{P K}$.

The security model of CombAS can be defined by following the security models of SeqAS and SyncAS. In the security model of CombAS, the public parameters and the challenge public key are given to the adversary at first. After that the adversary can request various queries for sequential aggregation and synchronized aggregation. Finally, the adversary outputs a forged aggregate signature which is sequential aggregation or synchronized aggregation. The adversary breaks the CombAS scheme if the forged aggregate signature is valid and it is non-trivial.

Definition 5.2 (Unforgeability). The security notion of existential unforgeability under a chosen message attack is defined in terms of the following experiment between a challenger $\mathcal{C}$ and a PPT adversary $\mathcal{A}$ :

1. Setup: $\mathcal{C}$ first initializes a key-pair list KeyList as empty. Next, it runs Setup to obtain public parameters $P P$ and KeyGen to obtain a key pair $(P K, S K)$, and gives $P K$ to $\mathcal{A}$.

2. Certification Query: $\mathcal{A}$ adaptively requests the certification of a public key by providing a key pair $(P K, S K)$. Then $\mathcal{C}$ adds the key pair $(P K, S K)$ to KeyList if the key pair is a valid one.

3. Sequential Aggregate Signature Query: $\mathcal{A}$ adaptively requests a sequential aggregate signature (by providing an aggregate-so-far $\sigma_{\Sigma}^{\prime}$ on messages $\mathbf{M}^{\prime}$ under public keys $\left.\mathbf{P K}^{\prime}\right)$, on a message $M$ to sign under the challenge public key $P K$, and receives a sequential aggregate signature $\sigma_{\Sigma}$.

4. Signature Query: $\mathcal{A}$ adaptively requests a signature for synchronized aggregation on a message $M$ and a time period $w$ that was not used before to sign under the challenge public key $P K$, and receives an individual signature $\sigma$.

5. Output: Finally (after a sequence of the above queries), $\mathcal{A}$ outputs a forged (sequential or synchronized) aggregate signature $\sigma_{\Sigma}^{*}$ on messages $\mathbf{M}^{*}$ under public keys $\mathbf{P} \mathbf{K}^{*} . \mathcal{C}$ outputs 1 if the forged signature satisfies the following three conditions, or outputs 0 otherwise: 1) $\mathbf{A g g V e r i f y}\left(\sigma_{\Sigma}^{*}, \mathbf{M}^{*}, \mathbf{P K} \mathbf{K}^{*}, P P\right)=$ 1, 2) The challenge public key $P K$ must exist in $\mathbf{P} \mathbf{K}^{*}$ and each public key in $\mathbf{P} \mathbf{K}^{*}$ except the challenge public key must be in KeyList, and 3) If the type of $\sigma_{\Sigma}^{*}$ is sequential aggregation, then the corresponding message $M$ in $\mathbf{M}^{*}$ of the challenge public key PK must not have been queried by $\mathcal{A}$ to the sequential aggregate signing oracle. If the type of $\sigma_{\Sigma}^{*}$ is synchronized aggregation, then the corresponding message $M$ in $\mathbf{M}^{*}$ of the challenge public key PK must not have been queried by $\mathcal{A}$ to the signing oracle for synchronized aggregation.

The advantage of $\mathcal{A}$ is defined as $\boldsymbol{A} \boldsymbol{d v}_{\mathcal{A}}^{\text {CombAS }}=\operatorname{Pr}[\mathcal{C}=1]$ where the probability is taken over all the randomness of the experiment. A SyncAS scheme is existentially unforgeable under a chosen message attack if all PPT adversaries have at most a negligible advantage (for large enough security parameter) in the above experiment. 


\subsection{Construction}

A simple CombAS scheme can be constructed by using a SeqAS scheme and a SyncAS scheme independently. However, the demerit of this simple CombAS scheme is that the size of public key and private key increases and the overload of certificate management for each public keys also increases. To solve these problems, we propose an efficient CombAS scheme by combining our SeqAS scheme and our SyncAS scheme.

Let $\mathcal{W}$ be a set of time periods where $|\mathcal{W}|$ is fixed polynomial in the security parameter. Our CombAS scheme is described as follows:

CombAS.Setup $\left(1^{\lambda}\right)$ : This algorithm first generates the bilinear groups $\mathbb{G}, \mathbb{G}_{T}$ of prime order $p$ of bit size $\Theta(\lambda)$. Let $g$ be the generator of $\mathbb{G}$. It chooses a random element $Y \in \mathbb{G}$ and two hash functions $H_{1}:\{0,1\} \times \mathcal{W} \rightarrow \mathbb{G}$ and $H_{2}:\{0,1\}^{*} \times \mathcal{W} \rightarrow \mathbb{Z}_{p}^{*}$. It outputs public parameters as $P P=$ $\left(p, \mathbb{G}, \mathbb{G}_{T}, e, g, Y, H_{1}, H_{2}\right)$. Note that the public parameters of our SeqAS scheme $P P_{\text {seq }}$ and the public parameters of our SyncAS scheme $P P_{s y n c}$ can be easily derived from $P P$.

CombAS.KeyGen $(P P)$ : This algorithm takes as input the public parameters $P P$. It selects a random exponent $x \in \mathbb{Z}_{p}$ and sets $X=g^{x}$. Then it outputs a private key as $S K=x$ and a public key as $P K=X$.

CombAS.AggSign $\left(\sigma_{\Sigma}^{\prime}, \mathbf{M}^{\prime}, \mathbf{P K} \mathbf{K}^{\prime}, M, S K, P P\right)$ : This algorithm first checks that the type of $\sigma_{\Sigma}^{\prime}$ is sequential aggregation, and then it outputs a sequential aggregate signature $\sigma_{\Sigma}$ by running $\operatorname{SeqAS.AggSign}\left(\sigma_{\Sigma}^{\prime}, \mathbf{M}^{\prime}\right.$, $\left.\mathbf{P K}^{\prime}, M, S K, P P_{\text {seq }}\right)$. Note that the signature implicitly includes the type of aggregation.

$\operatorname{CombAS.Sign}(M, w, S K, P P)$ : This algorithm outputs a individual signature $\sigma$ for synchronized aggregation by running $\operatorname{SyncAS.Sign}\left(M, w, S K, P P_{\text {sync }}\right)$. Note that the signature implicitly includes the type of aggregation.

CombAS.Verify $(\sigma, M, P K, P P)$ : This algorithm first checks that the type of $\sigma$ is synchronized aggregation, and then it outputs SyncAS.Verify $\left(\sigma, M, P K, P P_{\text {sync }}\right)$.

CombAS.Aggregate(S, M, PK, $P P)$ : This algorithm first checks that the type of signatures $\mathbf{S}$ is synchronized aggregation, and then it outputs an aggregate signature $\sigma_{\Sigma}$ for synchronized aggregation by running SyncAS.Aggregate $\left(\mathbf{S}, \mathbf{M}, \mathbf{P K}, P P_{\text {sync }}\right)$. Note that the signature implicitly includes the type of aggregation.

CombAS.AggVerify $\left(\sigma_{\Sigma}, \mathbf{M}, \mathbf{P K}, P P\right)$ : This algorithm first checks that the type of $\sigma_{\Sigma}$ is sequential or synchronized aggregation. If it is sequential aggregation, then it outputs $\operatorname{SeqAS.AggVerify}\left(\sigma_{\Sigma}, \mathbf{M}, \mathbf{P K}, P P_{\text {seq }}\right)$. Otherwise, it outputs SyncAS.AggVerify $\left(\sigma_{\Sigma}, \mathbf{M}, \mathbf{P K}, P P_{\text {sync }}\right)$.

\subsection{Security Analysis}

We prove the security of our CombAS scheme based on the security of our SeqAS scheme and our SyncAS scheme.

Theorem 5.3. The above CombAS scheme is existentially unforgeable under a chosen message attack if the SeqAS scheme and SyncAS scheme are existentially unforgeable under a chosen message attack. That is, for any PPT adversary $\mathcal{A}$ for the above CombAS scheme, there exists a PPT algorithm $\mathcal{B}$ for the $C L$ signature scheme such that $\boldsymbol{A d} \boldsymbol{v}_{\mathcal{A}}^{\text {CombAS }}(\lambda) \leq \boldsymbol{A} \boldsymbol{d} \boldsymbol{v}_{\mathcal{B}}^{\operatorname{SeqAS}}(\lambda)+\boldsymbol{A} \boldsymbol{d} \boldsymbol{v}_{\mathcal{B}}^{\text {SyncAS }}(\lambda)$. 
Proof. To prove the security of the CombAS scheme, we divide the behavior of an adversary as two types: Type-I and Type-II. The Type-I adversary outputs a forged sequential aggregate signature, but the Type-II adversary outputs a forged synchronized aggregate signature. Let $T_{I}, T_{I I}$ be the event such that an adversary behave like the Type-I, Type-II adversary respectively. In Lemma 5.4, we show that a Type-I adversary can be used for breaking the SeqAS scheme. In Lemma 5.5, we show that a Type-II adversary can be used for breaking the SyncAS scheme too. Therefore we have

$$
\begin{aligned}
\operatorname{Adv}_{\mathcal{A}}^{\operatorname{CombAS}}(\lambda) & \leq \operatorname{Pr}\left[T_{I}\right] \cdot \mathbf{A d v}_{\mathcal{A}_{I}}^{\operatorname{CombAS}}(\lambda)+\operatorname{Pr}\left[T_{I I}\right] \cdot \mathbf{A d v}_{\mathcal{A}_{I I}}^{\text {CombAS }}(\lambda) \\
& \leq \operatorname{Pr}\left[T_{I}\right] \cdot \mathbf{A d v}_{\mathcal{B}}^{\operatorname{SeqAS}}(\lambda)+\left(1-\operatorname{Pr}\left[T_{I}\right]\right) \cdot \mathbf{A d v}_{\mathcal{B}}^{\text {SyncAS }}(\lambda) \\
& \leq \mathbf{A d v}_{\mathcal{B}}^{\operatorname{SeqAS}}(\boldsymbol{\lambda})+\mathbf{A d v}_{\mathcal{B}}^{\text {SyncAS }}(\lambda)
\end{aligned}
$$

This completes our proof.

Lemma 5.4. If there is a Type-I adversary $\mathcal{A}_{I}$ that forges the above CombAS scheme, then there is a PPT algorithm $\mathcal{B}$ that forges our SeqAS scheme.

Proof. Suppose there exists an adversary $\mathcal{A}_{I}$ that forges the above CombAS scheme with non-negligible advantage $\varepsilon$. A simulator $\mathcal{B}$ that forges our SeqAS scheme is first given: the public parameters $P P_{\text {seq }}=$ $\left(p, \mathbb{G}, \mathbb{G}_{T}, e, g, Y\right)$ and a challenge public key $P K_{\text {seq }}=X$. Then $\mathcal{B}$ that interacts with $\mathcal{A}_{I}$ is described as follows:

Setup: $\mathcal{B}$ first constructs $P P=\left(p, \mathbb{G}, \mathbb{G}_{T}, e, g, Y, H_{1}, H_{2}\right)$ and $P K^{*}=X$ from $P P_{\text {seq }}$ and $P K_{\text {seq }}$. It initializes a key-pair list KeyList, hash lists $H_{1}$-List,$H_{2}$-List as an empty one and gives $P P$ and $P K^{*}$ to $\mathcal{A}_{I}$.

Certification Query: If $\mathcal{A}_{I}$ adaptively requests the certification of a public key, then $\mathcal{B}$ queries to its own certification oracle.

Hash Query: If $\mathcal{A}_{I}$ requests a $H_{1}$ hash query on a bit $b \in\{0,1\}$ and a time period $w_{i}$, then $\mathcal{B}$ selects a random exponent $r_{b, i} \in \mathbb{Z}_{p}$ and sets $H_{1}\left(b|| w_{i}\right)=g^{r_{b, i}}$. If $\mathcal{A}_{I}$ requests a $H_{2}$ hash query on a message $M_{i}$ and a time period $w_{j}$, then $\mathcal{B}$ selects a random value $h_{i, j} \in \mathbb{Z}_{p}$ and sets $H_{2}\left(M_{i}|| w_{j}\right)=h_{i, j}$. Note that $\mathcal{B}$ keeps the tuple $\left(b, w_{i}, r_{b, i}, H_{1}\left(b_{i} \| w_{i}\right)\right)$ in $H_{1}$-List and the tuple $\left(M_{i}, w_{j}, h_{i, j}\right)$ in $H_{2}$-List.

Sequential Aggregate Signature Query: If $\mathcal{A}$ adaptively requests a sequential aggregate signature, then $\mathcal{B}$ queries to its own sequential aggregate signature oracle.

Signature Query: If $\mathcal{A}_{I}$ adaptively requests a signature for synchronized aggregation by providing a message $M_{i}$ and a time period $w_{j}$ to sign under the challenge private key of $P K^{*}$, then $\mathcal{B}$ responds $\sigma_{i, j}=$ $\left(X^{r_{0, i}} X^{r_{1, i}} h_{i, j}, w_{j}\right)$ where $r_{0, i}, r_{1, i}$, and $h_{i, j}$ are retrieved from the $H_{1}$-List and $H_{2}$-List.

Output: $\mathcal{A}_{I}$ outputs a forged sequential aggregate signature $\sigma_{\Sigma}^{*}$ on messages $\mathbf{M}^{*}$ under public keys $\mathbf{P K}{ }^{*}$. $\mathcal{B}$ outputs $\sigma_{\Sigma}^{*}$ as the forged sequential aggregate signature for the SeqAS scheme.

To finish the proof, we only need to show that the distribution of hash outputs and signatures for synchronized aggregation is correct. It is obvious that the outputs of hash functions and the signatures for synchronized aggregation are correctly distributed. This completes our proof.

Lemma 5.5. If there is a Type-II adversary $\mathcal{A}_{I I}$ that forges the above CombAS scheme, then there is a PPT algorithm $\mathcal{B}$ that forges our SyncAS scheme.

Proof. Suppose there exists an adversary $\mathcal{A}_{I I}$ that forges the above CombAS scheme with non-negligible advantage $\varepsilon$. A simulator $\mathcal{B}$ that forges the SyncAS scheme is first given: the public parameters $P K_{\text {sync }}=$ $\left(p, \mathbb{G}, \mathbb{G}_{T}, e, g, H_{1}, H_{2}\right)$ and a challenge public key $P K_{s y n c}=X$. Then $\mathcal{B}$ that interacts with $\mathcal{A}_{I I}$ is described as follows: 
Setup: $\mathcal{B}$ first selects a random exponent $y \in \mathbb{Z}_{p}$, and then it sets $P P=\left(p, \mathbb{G}, \mathbb{G}_{T}, e, g, Y=g^{y}, H_{1}, H_{2}\right)$ and $P K^{*}=X$ from $P P_{\text {sync }}$ and $P K_{\text {sync }}$.

Certification Query: If $\mathcal{A}_{I I}$ requests the certification of a public key, then $\mathcal{B}$ queries to its own certification oracle.

Hash Query: If $\mathcal{A}_{I I}$ requests the hash values, then $\mathcal{B}$ queries to its own hash oracles.

Sequential Aggregate Signature Query: $\mathcal{A}_{I I}$ adaptively requests a sequential aggregate signature by providing an aggregate-so-far $\sigma_{\Sigma}^{\prime}$ on messages $\mathbf{M}^{\prime}=\left(M_{1}, \ldots, M_{k}\right)$ under public keys $\mathbf{P} \mathbf{K}^{\prime}=\left(P K_{1}, \ldots, P K_{k}\right)$, and a message $M$ to sign under the challenge private key of $P K^{*} . \mathcal{B}$ proceeds this query as follows:

1. It first checks that the signature $\sigma_{\Sigma}^{\prime}$ is valid by calling CombAS.AggVerify and that each public key in $\mathbf{P K}^{\prime}$ exits in KeyList.

2. It selects a random exponent $r \in \mathbb{Z}_{p}$ and creates a signature on the message $M$ for the challenge public key $P K^{*}=X$ as $\sigma=\left(A=g^{r}, B=Y^{r}, C=X^{r} \cdot X^{r y M}\right)$.

3. For each $1 \leq i \leq k$, it builds an aggregate signature on message $M_{i}$ using CombAS.AggSign since it knows the private key that corresponds to $P K_{i}$. The resulting signature is an aggregate signature for messages $\mathbf{M}^{\prime}|| M$ under public keys $\mathbf{P} \mathbf{K}^{\prime}|| P K^{*}$ since this scheme does not check the order of aggregation. It gives the result signature $\sigma_{\Sigma}$ to $\mathcal{A}_{I I}$.

Signature Query: If $\mathcal{A}_{I I}$ requests a signature for synchronized aggregation, then $\mathcal{B}$ queries to its own signature oracle.

Output: $\mathcal{A}_{I I}$ outputs a forged synchronized aggregate signature $\sigma_{\Sigma}^{*}$ on messages $\mathbf{M}^{*}$ under public keys $\mathbf{P K}$. $\mathcal{B}$ outputs $\sigma_{\Sigma}^{*}$ as the forged synchronized aggregate signature for the SyncAS scheme.

To finish the proof, we only need to show that the distribution of sequential aggregate signatures is correct. It is easy to check that the distribution of the signature $\sigma$ for the challenge public key is correct since $X^{r} \cdot X^{r y M}=\left(g^{r}\right)^{x}\left(Y^{r}\right)^{x M}=A^{x} B^{x M}$. This completes our proof.

\section{Conclusion}

In this paper we concentrated on the notion of aggregate signatures which applications are in reducing space of signatures for large repositories (such as in the legal, financial, and infrastructure areas). We first proposed a new sequential aggregate signature scheme and a new synchronized aggregate signature scheme using a newly devised "public key sharing" technique, and we proved their security under the security of the CL signature scheme. We also introduced a new notion of aggregate signature named combined aggregate signature and proposed an efficient construction based on the CL signature. Our aggregate signature schemes in this paper sufficiently satisfy the efficiency properties of aggregate signatures such that the size of public keys should be short, the size of aggregate signatures should be short, and the aggregate verification should be efficient.

An interesting problem is to prove the security of our SeqAS scheme under static assumptions instead of the interactive LRSW assumption. Recently, Gerbush et al. [23] proposed a modified CL signature scheme in bilinear groups of composite order and proved its security under static assumptions. One may consider to use the modified CL signature scheme of Gerbush et al. for aggregate signature schemes, but it is not easy to apply our techniques to their modified CL signature scheme. 


\section{Acknowledgements}

We thank the anonymous reviewers of FC 2013 for their valuable comments.

\section{References}

[1] Jae Hyun Ahn, Matthew Green, and Susan Hohenberger. Synchronized aggregate signatures: new definitions, constructions and applications. In ACM Conference on Computer and Communications Security, pages 473-484, 2010.

[2] Giuseppe Ateniese, Jan Camenisch, and Breno de Medeiros. Untraceable rfid tags via insubvertible encryption. In Vijay Atluri, Catherine Meadows, and Ari Juels, editors, ACM Conference on Computer and Communications Security, pages 92-101. ACM, 2005.

[3] Giuseppe Ateniese, Jan Camenisch, Susan Hohenberger, and Breno de Medeiros. Practical group signatures without random oracles. Cryptology ePrint Archive, Report 2005/385, 2005. http:// eprint.iacr.org/2005/385.

[4] Ali Bagherzandi and Stanislaw Jarecki. Identity-based aggregate and multi-signature schemes based on rsa. In Phong Q. Nguyen and David Pointcheval, editors, Public Key Cryptography, volume 6056 of Lecture Notes in Computer Science, pages 480-498. Springer, 2010.

[5] Mihir Bellare, Chanathip Namprempre, and Gregory Neven. Unrestricted aggregate signatures. In Lars Arge, Christian Cachin, Tomasz Jurdzinski, and Andrzej Tarlecki, editors, ICALP, volume 4596 of Lecture Notes in Computer Science, pages 411-422. Springer, 2007.

[6] Mihir Bellare and Gregory Neven. Identity-based multi-signatures from rsa. In Masayuki Abe, editor, CT-RSA, volume 4377 of Lecture Notes in Computer Science, pages 145-162. Springer, 2007.

[7] Adam Bender, Jonathan Katz, and Ruggero Morselli. Ring signatures: Stronger definitions, and constructions without random oracles. J. Cryptology, 22(1):114-138, 2009.

[8] Alexandra Boldyreva, Craig Gentry, Adam O’Neill, and Dae Hyun Yum. Ordered multisignatures and identity-based sequential aggregate signatures, with applications to secure routing. In Peng Ning, Sabrina De Capitani di Vimercati, and Paul F. Syverson, editors, ACM Conference on Computer and Communications Security, pages 276-285. ACM, 2007.

[9] Alexandra Boldyreva, Craig Gentry, Adam O’Neill, and Dae Hyun Yum. Ordered multisignatures and identity-based sequential aggregate signatures, with applications to secure routing. Cryptology ePrint Archive, Report 2007/438, 2010. http: / /eprint.iacr.org/2007/438.

[10] Alexandra Boldyreva, Adriana Palacio, and Bogdan Warinschi. Secure proxy signature schemes for delegation of signing rights. J. Cryptology, 25(1):57-115, 2012.

[11] Dan Boneh and Xavier Boyen. Short signatures without random oracles. In Christian Cachin and Jan Camenisch, editors, EUROCRYPT, volume 3027 of Lecture Notes in Computer Science, pages 56-73. Springer, 2004.

[12] Dan Boneh and Matthew K. Franklin. Identity-based encryption from the weil pairing. In Joe Kilian, editor, CRYPTO, volume 2139 of Lecture Notes in Computer Science, pages 213-229. Springer, 2001. 
[13] Dan Boneh, Craig Gentry, Ben Lynn, and Hovav Shacham. Aggregate and verifiably encrypted signatures from bilinear maps. In Eli Biham, editor, EUROCRYPT, volume 2656 of Lecture Notes in Computer Science, pages 416-432. Springer, 2003.

[14] Dan Boneh, Ben Lynn, and Hovav Shacham. Short signatures from the weil pairing. In Colin Boyd, editor, ASIACRYPT, volume 2248 of Lecture Notes in Computer Science, pages 514-532. Springer, 2001.

[15] Kyle Brogle, Sharon Goldberg, and Leonid Reyzin. Sequential aggregate signatures with lazy verification from trapdoor permutations - (extended abstract). In Xiaoyun Wang and Kazue Sako, editors, ASIACRYPT, volume 7658 of Lecture Notes in Computer Science, pages 644-662. Springer, 2012.

[16] Jan Camenisch, Susan Hohenberger, and Michael Østergaard Pedersen. Batch verification of short signatures. In Moni Naor, editor, EUROCRYPT, volume 4515 of Lecture Notes in Computer Science, pages 246-263. Springer, 2007.

[17] Jan Camenisch and Anna Lysyanskaya. Signature schemes and anonymous credentials from bilinear maps. In Matthew K. Franklin, editor, CRYPTO, volume 3152 of Lecture Notes in Computer Science, pages 56-72. Springer, 2004.

[18] Ran Canetti, Shai Halevi, and Jonathan Katz. A forward-secure public-key encryption scheme. In Eli Biham, editor, EUROCRYPT, volume 2656 of Lecture Notes in Computer Science, pages 255-271. Springer, 2003.

[19] Ran Canetti, Shai Halevi, and Jonathan Katz. A forward-secure public-key encryption scheme. J. Cryptology, 20(3):265-294, 2007.

[20] Marc Fischlin, Anja Lehmann, and Dominique Schröder. History-free sequential aggregate signatures. In Ivan Visconti and Roberto De Prisco, editors, SCN, volume 7485 of Lecture Notes in Computer Science, pages 113-130. Springer, 2012.

[21] Sanjam Garg, Craig Gentry, and Shai Halevi. Candidate multilinear maps from ideal lattices. In Thomas Johansson and Phong Q. Nguyen, editors, EUROCRYPT, volume 7881 of Lecture Notes in Computer Science, pages 1-17. Springer, 2013.

[22] Craig Gentry and Zulfikar Ramzan. Identity-based aggregate signatures. In Moti Yung, Yevgeniy Dodis, Aggelos Kiayias, and Tal Malkin, editors, Public Key Cryptography, volume 3958 of Lecture Notes in Computer Science, pages 257-273. Springer, 2006.

[23] Michael Gerbush, Allison B. Lewko, Adam O’Neill, and Brent Waters. Dual form signatures: An approach for proving security from static assumptions. In Xiaoyun Wang and Kazue Sako, editors, ASIACRYPT, volume 7658 of Lecture Notes in Computer Science, pages 25-42. Springer, 2012.

[24] Susan Hohenberger, Amit Sahai, and Brent Waters. Full domain hash from (leveled) multilinear maps and identity-based aggregate signatures. In Ran Canetti and Juan A. Garay, editors, CRYPTO (1), volume 8042 of Lecture Notes in Computer Science, pages 494-512. Springer, 2013.

[25] Kwangsu Lee, Dong Hoon Lee, and Moti Yung. Aggregating cl-signatures revisited: Extended functionality and better efficiency. In Ahmad-Reza Sadeghi, editor, Financial Cryptography, volume 7859 of Lecture Notes in Computer Science, pages 171-188. Springer, 2013. 
[26] Kwangsu Lee, Dong Hoon Lee, and Moti Yung. Sequential aggregate signatures made shorter. In Michael J. Jacobson Jr., Michael E. Locasto, Payman Mohassel, and Reihaneh Safavi-Naini, editors, ACNS, volume 7954 of Lecture Notes in Computer Science, pages 202-217. Springer, 2013.

[27] Kwangsu Lee, Dong Hoon Lee, and Moti Yung. Sequential aggregate signatures with short public keys: Design, analysis and implementation studies. In Kaoru Kurosawa and Goichiro Hanaoka, editors, Public Key Cryptography, volume 7778 of Lecture Notes in Computer Science, pages 423-442. Springer, 2013.

[28] Steve Lu, Rafail Ostrovsky, Amit Sahai, Hovav Shacham, and Brent Waters. Sequential aggregate signatures and multisignatures without random oracles. In Serge Vaudenay, editor, EUROCRYPT, volume 4004 of Lecture Notes in Computer Science, pages 465-485. Springer, 2006.

[29] Anna Lysyanskaya, Silvio Micali, Leonid Reyzin, and Hovav Shacham. Sequential aggregate signatures from trapdoor permutations. In Christian Cachin and Jan Camenisch, editors, EUROCRYPT, volume 3027 of Lecture Notes in Computer Science, pages 74-90. Springer, 2004.

[30] Anna Lysyanskaya, Ronald L. Rivest, Amit Sahai, and Stefan Wolf. Pseudonym systems. In Howard M. Heys and Carlisle M. Adams, editors, Selected Areas in Cryptography, volume 1758 of Lecture Notes in Computer Science, pages 184-199. Springer, 1999.

[31] Moni Naor and Moti Yung. Universal one-way hash functions and their cryptographic applications. In David S. Johnson, editor, STOC, pages 33-43. ACM, 1989.

[32] Gregory Neven. Efficient sequential aggregate signed data. In Nigel P. Smart, editor, EUROCRYPT, volume 4965 of Lecture Notes in Computer Science, pages 52-69. Springer, 2008.

[33] Dominique Schröder. How to aggregate the cl signature scheme. In Vijay Atluri and Claudia Díaz, editors, ESORICS, volume 6879 of Lecture Notes in Computer Science, pages 298-314. Springer, 2011.

[34] Victor Shoup. Lower bounds for discrete logarithms and related problems. In Walter Fumy, editor, EUROCRYPT, volume 1233 of Lecture Notes in Computer Science, pages 256-266. Springer, 1997.

[35] Brent Waters. Efficient identity-based encryption without random oracles. In Ronald Cramer, editor, EUROCRYPT, volume 3494 of Lecture Notes in Computer Science, pages 114-127. Springer, 2005. 